\title{
Preferences Single-Peaked on a Circle
}

\author{
Dominik Peters \\ Carnegie Mellon University \\ Computer Science Department \\ 5000 Forbes Avenue, Pittsburgh, PA 15213, USA \\ Martin Lackner \\ TU Wien \\ Databases and Artificial Intelligence Group \\ Favoritenstraße 9-11, 1040 Vienna, Austria
}

\begin{abstract}
We introduce the domain of preferences that are single-peaked on a circle, which is a generalization of the well-studied single-peaked domain. This preference restriction is useful, e.g., for scheduling decisions, certain facility location problems, and for one-dimensional decisions in the presence of extremist preferences. We give a fast recognition algorithm of this domain, provide a characterisation by finitely many forbidden subprofiles, and show that many popular single- and multi-winner voting rules are polynomial-time computable on this domain. In particular, we prove that Proportional Approval Voting can be computed in polynomial time for profiles that are single-peaked on a circle. In contrast, Kemeny's rule remains hard to evaluate, and several impossibility results from social choice theory can be proved using only profiles in this domain.
\end{abstract}

\section{Introduction}

A central problem in the study of multi-agent systems is the aggregation of agents' preferences in order to make group decisions. Impossibility theorems and computational hardness result make this problem a hard one to solve. However, a successful line of research going back to Black's (1948) seminal article has managed to circumvent many problems in (computational) social choice for the special case when agents' preferences are single-peaked. Under this preference restriction, we assume that agents have preferences over the possible values of a one-dimensional quantity such as the timing of a deadline, a tax rate, a thermostat setting, or the price of a new product. A preference ordering is single-peaked if an agent has a certain most-preferred value of the quantity and derives less and less satisfaction from values that are further away from the subjective optimum. Another popular application of this setting is in political elections, where it is often held that candidates can be ordered on a left-to-right spectrum making the voters' preferences single-peaked.

Preference profiles that consist solely of single-peaked preference orderings have attractive properties, both algorithmically and in terms of their social choice behaviour (Elkind, Lackner, \& Peters, 2016, 2017). For example, winner determination problems that are computationally hard in the general case tend to be easy when restricted to single-peaked profiles (Betzler, Slinko, \& Uhlmann, 2013; Brandt, Brill, Hemaspaandra, \& Hemaspaandra, 2015), and the single-peaked domain guarantees the existence of Condorcet winners 


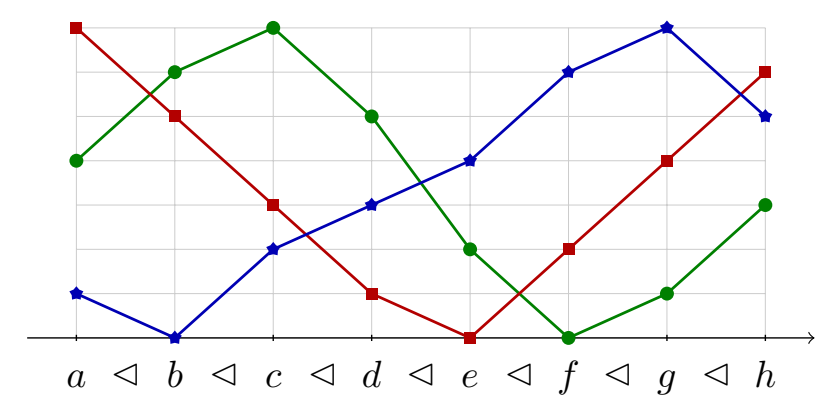

Figure 1: Example of preferences single-peaked on a circle.

as well as transitivity of the majority relation and thus admits a strategyproof voting rule (Moulin, 1991).

The usefulness of results of this type is limited by the extent to which profiles in practice actually happen to be single-peaked. One way of dealing with this is to consider less restrictive generalisations of single-peakedness. Maybe the structure of the alternative space is not quite one-dimensional, and in this case it might be useful to consider preferences that are single-peaked on a tree (Demange, 1982). This domain is notably larger, yet still retains many desirable properties in social choice terms; however, its algorithmic usefulness is more mixed (Yu, Chan, \& Elkind, 2013; Peters \& Elkind, 2016).

In this paper, we identify a new preference restriction: being single-peaked on a circle. Here we assume that alternatives can be placed on a circle, with agents' preferences again being decreasing on both sides of their peaks. See Figure 1 for some example shapes that 'preference curves' might have in this setting; higher points are more preferred. Note that the circle wraps around, and so $h$ and $a$ are adjacent. Intuitively, a preference profile is single-peaked on a circle if, for every agent, we can 'cut' the cycle once so that the agent's preferences are single-peaked on the resulting line. Crucially, the location of the cutting point may differ for each agent.

The aim of this paper is to explore this new preference domain in detail. We will find that this domain is algorithmically useful (it often allows for efficient winner determination), but it performs less convincingly in terms of axiomatic properties (since voting paradoxes still occur and impossibility results can still be proven). Interestingly, this is precisely opposite to how the results turned out for single-peakedness on trees.

\subsection{Motivating Examples}

There are many practical scenarios where we might expect preferences to be single-peaked on a circle. This is even the case when, on first sight, there seems to be no circle anywhere. Indeed, suppose that alternatives are naturally ordered on a line; we may pretend this line is a circle by joining up its endpoints. Of course, every order that is single-peaked on the line is also single-peaked on the circle.

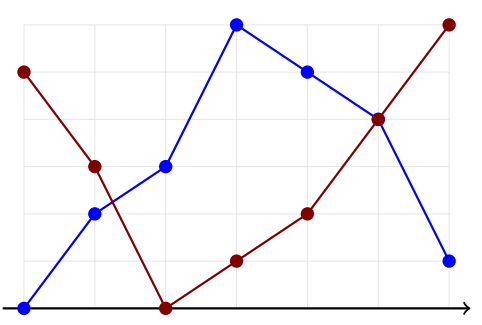
But crucially, the reverse of such an order, now single-caved (also known as single-dipped) on the line, is still single-peaked on the same circle. Thus, our new preference restriction 
allows us to combine single-peaked and single-caved votes (as shown on the right). One interpretation is that this move allows us to accommodate "extremists". For example, while most people have a sweet spot somewhere on the left-right political axis, some people might dislike centrist options and prefer the extremes. Indeed, an analysis of Tangian (2020) shows that the parties participating in the 2017 Bundestag elections in Germany can be sensibly aligned on a left-right axis if this axis is rolled into a "horsehoe-like" form, i.e., in a circular arrangement. On a lighter note, when planning a vacation, some might have an optimal climate in mind, while others like it both very cold (skiing) and very hot (beaches), but dislike compromises (England).

Other examples of alternative spaces are more explicitly cyclic. Consider, for example, finding a good time for a daily event (such as a day or night shift, or a meeting, or the timing of backups) where possibilities are arranged in a 24-hour cycle. A similar structure exists when scheduling an international phone call; here, different time zones are arranged along the equator, and lead to cyclic preferences.

But perhaps the most appealing example of preferences that can be expected to be single-peaked on a circle come from problems inspired by facility location. Rather many structures have a boundary that is (roughly) isomorphic to a cycle, including most cities and countries. The problem of deciding where to locate a new airport for a city would be one example, since airports are usually positioned on the boundary. Similarly, where should a company build new office space? To which coastal region should a family move? Where do we want to sit in a football stadium? Another plausible application could be inspired by security concerns, if we consider the placement of border security checkpoints.

\subsection{Contributions}

The main contributions of this paper can be summarised as follows:

- We formally define single-peakedness on circles and immediately extend this definition to preferences with ties, and to dichotomous (approval) preferences. Thus, our proposed domain is strictly larger than the class of possibly single-peaked preferences (Lackner, 2014; Fitzsimmons \& Lackner, 2020) and candidate interval dichotomous preferences (Faliszewski, Hemaspaandra, Hemaspaandra, \& Rothe, 2011; Elkind \& Lackner, 2015).

- We show that it is possible to efficiently recognise whether a given preference profile is single-peaked on some circle, and if so return a suitable circle. For the case of preferences without ties, we give a recognition algorithm that runs in linear time, matching the performance in the case for the line.

- We characterise the domain of preferences single-peaked on a circle through a list of finitely many forbidden subprofiles with 2 voters and 5 alternatives, and with 3 voters and 4 alternatives. The proof of this characterisation implies that our lineartime recognition algorithm can certify its negative answers by exhibiting a forbidden subprofile.

- While single-peakedness on a line serves as a way to circumvent many impossibility results in social choice, we show that such impossibilities (including the famous im- 
possibility result by Gibbard and Satterthwaite) can still be proven when preferences are allowed to be single-peaked on a circle.

- We then study the algorithmic properties of our new preference extension. We show that Young's voting rule (and also Young scores) can be efficiently computed if preferences are single-peaked on a circle; this algorithm also improves upon the state-ofthe-art when it comes to preferences single-peaked on a line. In contrast, we show that Kemeny's rule is NP-hard to compute even in this restricted domain.

- Finally, we show that several multi-winner voting rules are efficiently computable in our restricted case, specifically all that are included in the large class of so-called OWAbased rules. This class includes, e.g., the Chamberlin-Courant rule and Proportional Approval Voting (PAV). It is noteworthy that some of these algorithmic results have not yet been established even for single-peaked profiles (such as the one for PAV). This general result relies on using total unimodularity and integer programming.

\subsection{Outline of the Paper}

In Section 2, we introduce and define single-peakedness on a circle. In Section 3, we discuss the algorithmic recognition of these preferences; proof details are delegated to the appendix, Section A. Building on these results, we prove a characterization of this preference class in Section 4, with proof details in the appendix, Section B. We then take the perspective of social choice theory in Section 5 and revisit classical impossibility results. The algorithmic usefulness of preferences that are single-peaked on a circle is the focus of the following two sections: Section 6 for the single-winner rules Kemeny and Young, and Section 7 for several multi-winner rules including Chamberlin-Courant and Proportional Approval Voting. Open problems are discussed in Section 8.

\section{Definition}

Let $A$ be a finite set of alternatives (or candidates). A weak order (or preference relation) is a binary relation $\succcurlyeq$ over $A$ which is complete and transitive. A linear order is a weak order that is antisymmetric, and so does not allow preference ties; a strict linear order $\succ$ is the irreflexive part of a linear order. A profile $P=\left(v_{1}, \ldots, v_{n}\right)$ over $A$ is a list of weak orders over $A$. The elements of $N=\{1, \ldots, n\}$ are called voters, and we associate voter $i \in N$ with the order $v_{i}$, which we call the vote of voter $i$. For convenience, we write $a \succcurlyeq_{i} b$ whenever $(a, b) \in v_{i}$, i.e., when voter $i$ weakly prefers alternative $a$ to alternative $b$. We also use $\succ_{i}$ and $\sim_{i}$ for the strict and indifference parts of $\succcurlyeq_{i}$. We will always write $m$ for the number of alternatives and $n$ for the number of voters. If $v_{i}$ is a linear order, we write $\operatorname{top}\left(v_{i}\right)$ for $i$ 's most-preferred alternative.

An axis $\triangleleft$ is a strict linear order of the alternatives. We usually think of an axis as describing the underlying one-dimensional structure of the alternative space. A linear order $v_{i}$ is single-peaked with respect to the axis $\triangleleft$ if for each pair of alternatives $a, b \in A$ with $\operatorname{top}\left(v_{i}\right) \triangleleft b \triangleleft a$ or $a \triangleleft b \triangleleft \operatorname{top}\left(v_{i}\right)$ it holds that $b \succ_{i} a$. Let us also give another, equivalent definition. An interval $I \subseteq A$ of an axis $\triangleleft$ is any set such that for all $a, b, c \in A$, if we have $a, c \in I$ and $a \triangleleft b \triangleleft c$, then $b \in I$. Then a vote $v_{i}$ is single-peaked with respect to the axis $\triangleleft$ if 
and only if for every $c \in A$, the top-initial segment $\left\{a \in A: a \succ_{i} c\right\}$ is an interval of $\triangleleft$. This definition in terms of intervals immediately gives a definition of the single-peaked property for weak orders as well. There are several possible definitions of single-peakedness for weak orders; the one stated above is sometimes known as possible single-peakedness (Lackner, 2014; Fitzsimmons \& Lackner, 2020), since it is equivalent to saying that there exists a linearisation of the weak order which is single-peaked.

We say that two axes $\triangleleft$ and $\triangleleft^{\prime}$ are cyclically equivalent if there is $l \in[m]$ such that we can write $a_{1} \triangleleft a_{2} \triangleleft a_{3} \triangleleft \cdots \triangleleft a_{m}$ and $a_{l} \triangleleft^{\prime} a_{l+1} \triangleleft^{\prime} \cdots \triangleleft^{\prime} a_{m} \triangleleft^{\prime} a_{1} \triangleleft^{\prime} \cdots \triangleleft^{\prime} a_{l-1}$. For an axis $\triangleleft$, we then define the circle $C(\triangleleft)$ of $\triangleleft$ to be the set of axes cyclically equivalent to $\triangleleft$. Any set $C$ of axes that can be written as $C=C(\triangleleft)$ for some $\triangleleft$ we call a circle. For example, $C=\left\{a \triangleleft b \triangleleft c, b \triangleleft^{\prime} c \triangleleft^{\prime} a, c \triangleleft^{\prime \prime} a \triangleleft^{\prime \prime} b\right\}$ is a circle. Note that "cutting" a circle $C$ at a point yields an axis $\triangleleft \in C$. We say that $\triangleleft$ starts in $a \in A$ if $a \triangleleft b$ for all $b \in A \backslash\{a\}$.

Definition. Let $C$ be a circle. $A$ vote $v_{i}$ is single-peaked on $C$ if there is an axis $\triangleleft \in C$ such that $v_{i}$ is single-peaked with respect to $\triangleleft$. A preference profile $P$ is single-peaked on a circle (SPOC) if there exists a circle $C$ such that every vote $v_{i} \in P$ is single-peaked on $C$.

Intuitively, a vote $v_{i}$ is single-peaked on $C$ if $C$ can be cut so that $v_{i}$ is single-peaked on the resulting line.

Again let us state another equivalent definition. An interval $I \subseteq A$ of a circle $C$ is a set that is an interval of one of the axes $\triangleleft \in C$ of the circle. Then a vote is single-peaked on a circle $C$ if and only if each top-initial segment $\left\{a \in A: a \succ_{i} c\right\}$ is an interval of $C$. Note that the complement $A \backslash I$ of an interval $I$ of $C$ is again an interval. Thus, a weak order $\succcurlyeq$ is

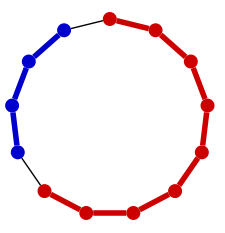

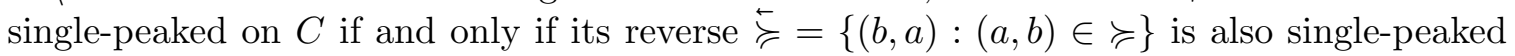
on $C$.

A vote is single-caved if its reverse is single-peaked. It follows, then, that mixtures of single-peaked and single-caved orders (on the same axis) are SPOC. However, not all SPOC profiles have this form; one such example is the profile shown in Figure 1, where the circle cannot be cut so as to make every preference curve either single-peaked or single-caved.

A weak order $\succcurlyeq$ is dichotomous if there is a partition of $A$ into sets $A_{1}$ and $A_{2}$ such that $a \succ b$ if and only if $a \in A_{1}$ and $b \in A_{2}$. A voter whose preferences are given by $\succcurlyeq$ is said to approve the alternatives in $A_{1}$. Note that, according to our definition, a profile of dichotomous (approval) preferences is SPOC if and only if there is a circle $C$ such that every voter's approval set is an interval of $C$.

\section{Recognition Algorithms}

In this section we design algorithms that decide whether a given profile is single-peaked on some circle, and if so, return a suitable circle $C$.

A matrix $M=\left(a_{i j}\right)$ with $a_{i j} \in\{0,1\}$ has the consecutive ones property if the columns of $M$ can be put into a linear order $\triangleleft$ so that for every row of $M$, the columns with 1-entries form an interval of $\triangleleft$. The matrix shown on the right is an example. The matrix $M$ has the circular ones property if its columns can be arranged in a circle $C$ so that the 1-entries of each row form an interval of $C$. Given our

$\left[\begin{array}{llllll}0 & 0 & 1 & 1 & 1 & 0 \\ 1 & 1 & 1 & 0 & 0 & 0 \\ 0 & 0 & 0 & 0 & 1 & 1 \\ 0 & 1 & 1 & 1 & 1 & 0 \\ 0 & 0 & 0 & 1 & 1 & 0\end{array}\right]$


definitions in terms of intervals above, it is straightforward to translate a profile $P$ of weak orders into an $m n \times m$ matrix $M$ so that $P$ is single-peaked [single-peaked on a circle] if and only if $M$ has the consecutive [circular] ones property (Bartholdi III \& Trick, 1986): Take one column for each alternative, and one row for every top-initial segment of every voter in $P$; the row is the incidence vector of the segment. Since it is possible to check in linear time whether a matrix $A$ has the consecutive or circular ones property (Booth \& Lueker, 1976), this gives us an $O\left(m^{2} n\right)$ algorithm to recognise profiles that are single-peaked on a circle.

In the remainder of this section, we design a more explicit algorithm that runs in time $O(m n)$ when the input profile consists of linear orders. ${ }^{1}$ The algorithm works by reducing the SPOC recognition problem for linear orders to the recognition problem of single-peaked profiles for weak orders, in such a way that we can apply an algorithm of Fitzsimmons and Lackner (2020).

Suppose $P=\left(v_{1}, \ldots, v_{n}\right)$ is a profile of linear orders over $A$, and fix some alternative $z \in A$. We will build another profile $\bar{P}=\left(v_{1}^{u}, v_{1}^{l}, \ldots, v_{n}^{u}, v_{n}^{l}\right)$ of $2 n$ weak orders by slicing each vote $v_{i}$ at $z$ into an upper part $v_{i}^{u}$ and a lower part $v_{i}^{l}$. The upper part $v_{i}^{u}$ ranks all alternatives $a$ such that $a \succ_{i} z$ in order of $\succ_{i}$, and puts all remaining alternatives into a least-preferred indifference class. The lower part $v_{i}^{l}$ ranks all alternatives $a$ such that $z \succ_{i} a$ in reverse order of $\succ_{i}$, and again puts all remaining alternatives into a least-preferred indifference class.

Example. Slicing the order $a \succ b \succ c \succ z \succ d \succ e \succ f$ at $z$ yields the upper part $a \succ^{u} b \succ^{u} c \succ^{u} z \sim^{u} d \sim^{u} e \sim^{u} f$ and the lower part $f \succ^{l} e \succ^{l} d \succ^{l} z \sim^{l} a \sim^{l} b \sim^{l} c$.

The notion of slicing reduces SPOC to single-peakedness:

Proposition 1. Suppose a profile $\bar{P}$ of weak orders is obtained by slicing each vote of a profile $P$ of linear orders at some fixed $z \in A$. Then $P$ is SPOC if and only if the profile $\bar{P}$ is single-peaked.

Proof. Suppose $P$ is SPOC on $C$, and let $\triangleleft \in C$ be an axis starting in $z$. Since $z$ is leastpreferred by all voters in $\bar{P}, z$ is not contained in any top-initial segment of any voter in $\bar{P}$. However, all top-initial segments of votes in $\bar{P}$ are intervals of $C$. Since they do not contain $z$, they must also be intervals of $\triangleleft$. Thus, $\bar{P}$ is single-peaked with respect to $\triangleleft$.

Suppose $\bar{P}$ is single-peaked with respect to $\triangleleft$. We show that $P$ is SPOC on $C=C(\triangleleft)$. Take a top-initial segment $S$ of a vote $v_{i}$ in $P$; we prove that $S$ is an interval of $C$. If $z \notin S$, then $S$ is a top-initial segment of $v_{i}^{u}$ in $\bar{P}$. Thus, $S$ is an interval of $\triangleleft$ and so an interval of $C$. If however $z \in S$, then the complement $A \backslash S$ is a top-initial segment of $v_{i}^{l}$ in $\bar{P}$, hence an interval of $\triangleleft$, and so $A \backslash S$ is an interval of $C$. But the complement of an interval of a circle is again an interval, and so $S$ is an interval of $C$. Hence $P$ is SPOC.

Thus, we can use an algorithm that decides whether a profile of weak orders is singlepeaked to decide whether a profile of linear orders is SPOC. Next, note that if we select $z \in A$ to be the alternative that is ranked last by $v_{1}$ (say), then the profile $\bar{P}$ obtained by slicing $P$ at $z$ contains a linear order (namely the upper part of $v_{1}$ ). Fitzsimmons and Lackner (2020) give an $O(m n)$ time algorithm that decides whether a profile of weak orders

1. Actually, the algorithm works whenever $P$ contains at least one linear order. 
containing at least one linear order is single-peaked. In Appendix A, for completeness, we include a description of the algorithm of Fitzsimmons and Lackner (2020), and the relevant parts of its correctnes proof.

Since $\bar{P}$ can be constructed from $P$ in time $O(m n)$, by Proposition 1 , we obtain the following.

Theorem 2. There is an $O(m n)$ time algorithm that decides whether a profile of linear orders is single-peaked on a circle.

\section{Characterisation by Forbidden Subprofiles}

Ballester and Haeringer (2011) have characterised the domain of single-peaked profiles of linear orders by a finite collection of forbidden subprofiles. More precisely, they gave forbidden profiles with 3 voters and 3 alternatives, and with 2 voters and 4 alternatives such that a profile $P$ is not single-peaked if and only if it is possible to obtain one of their forbidden profiles from $P$ by deleting and reordering voters, and deleting and renaming alternatives. A similar characterisation exists for single-crossing profiles (Bredereck, Chen, \& Woeginger, 2013), but no finite characterisation exists for $d$-Euclidean profiles (Chen, Pruhs, \& Woeginger, 2017; Peters, 2017).

Here, we prove that a profile is SPOC unless it contains certain forbidden subprofiles with 2 voters and 5 alternatives and with 3 voters and 4 alternatives. For sets $B, C \subseteq A$ of alternatives, let us write $B \succ_{i} C$ to mean that $b \succ_{i} c$ for all $b \in C$ and $c \in C$.

Theorem 3. A profile $P$ of linear orders on $A$ is not SPOC if and only if one of the following three cases occurs.

1. There are distinct alternatives $a, b, c, d, e \in A$ and voters $v_{i}$ and $v_{j}$ in $P$ such that

$$
\begin{aligned}
& \{a, b\} \succ_{i}\{c\} \succ_{i}\{d, e\}, \\
& \{a, e\} \succ_{j}\{c\} \succ_{j}\{d, b\} .
\end{aligned}
$$

2. There are distinct alternatives $a, b, c, d \in A$ and voters $v_{i}, v_{j}$, and $v_{k}$ in $P$ such that

$$
\begin{aligned}
& \{a, b\} \succ_{i}\{c, d\}, \\
& \{a, c\} \succ_{j}\{b, d\}, \\
& \{a, d\} \succ_{k}\{b, c\} .
\end{aligned}
$$

3. There are distinct alternatives $a, b, c, d \in A$ and voters $v_{i}, v_{j}$, and $v_{k}$ in $P$ such that

$$
\begin{aligned}
& \{a, b\} \succ_{i}\{c, d\}, \\
& \{b, c\} \succ_{j}\{a, d\}, \\
& \{c, a\} \succ_{k}\{b, d\} .
\end{aligned}
$$

Proof. Sufficiency. We prove that if one of the three cases occurs, then $P$ is not SPOC. Since SPOC is closed under alternative deletion, in each case, we may assume wlog that $P$ only involves alternatives mentioned in the forbidden condition. Suppose $P$ was single-peaked on the circle $C$. 
1. Considering top-initial segments of size 2 , we see that $a$ must have neighbours $b$ and $e$ in $C$. Considering top-initial segments of size 3 , and taking complements, we see that $d$ must have neighbours $b$ and $e$ in $C$. But this uniquely determines a circle with $a \triangleleft b \triangleleft d \triangleleft e \triangleleft a$; yet this circle does not include $c$, a contradiction.

2. Considering top-initial segments of size 2, we see that $a$ must have neighbours $b, c$, and $d$ in $C$. But no vertex of a circle has three neighbours, a contradiction.

3. Since $P$ is single-peaked on $C$, so is $P$ with every order reversed. But after reversing every order, we are again in case $2: d$ must have three neighbours.

Necessity. This direction is much more involved, and the full proof appears in Appendix B. The proof strategy is as follows: if $P$ is not SPOC, then the recognition algorithm of Theorem 2 , run on input $P$, will return a negative answer. We analyse every way the algorithm could answer negatively, and in each case construct a witnessing forbidden structure from among those identified in the theorem statement.

For the benefit of future research, let us briefly describe how we obtained Theorem 3 . We first implemented a recognition algorithm for SPOC profiles (Theorem 2) and then iterated through all possible profiles of certain sizes (up to isomorphism), checking for each whether they were minimal counterexamples: not SPOC, but every profile obtained by deleting a voter or an alternative is SPOC. We analysed the resulting list by hand to come up with the compact representation in Theorem 3. The proof strategy of case analysis of the "no"-conditions of a recognition algorithm is (implicitly) also the approach used in previous characterisations (Ballester \& Haeringer, 2011; Bredereck et al., 2013; Cornaz, Galand, \& Spanjaard, 2012).

\section{Impossibility Theorems}

One of the major advantages of the traditional single-peaked domain is the existence of a non-manipulable voting rule on this domain: The well-known median voter procedure sorts voters' most preferred alternatives according to the axis $\triangleleft$ and then returns the median alternative $a$. This alternative is, in fact, a (weak) Condorcet winner: for any other alternative $b$, a (weak) majority of voters prefers $a$ to $b$. One might hope to be able to extend this procedure to circles, but this turns out to be impossible: the Gibbard-Satterthwaite theorem can be proven using only profiles that are single-peaked on a circle.

A resolute voting rule $f$ on SPOC profiles is a function assigning a single winning alternative to every SPOC profile of linear orders. The rule $f$ is non-dictatorial if there is no fixed voter $i$ such that $f$ always picks $i$ 's top alternative. It is onto if for every alternative

$x$, there exists a SPOC profile $P$ with $f(P)=x$. The profile obtained from $P$ by replacing vote $v_{i}$ by $v_{i}^{\prime}$ is denoted by $\left(P_{-i}, v_{i}^{\prime}\right)$. A voting rule $f$ on SPOC profiles is strategyproof if $f(P) \succcurlyeq_{i} f\left(P_{-i}, v_{i}^{\prime}\right)$ for all orders $v_{i}^{\prime}$ such that $\left(P_{-i}, v_{i}^{\prime}\right)$ is still SPOC.

Theorem 4 (Gibbard-Satterthwaite Theorem for SPOC). There is no resolute voting rule on SPOC profiles that is non-dictatorial, onto, and satisfies strategyproofness. 
This follows immediately from the results of Kim and Roush (1980) and Sato (2010), who prove this result for an even more restricted domain consisting only of the $2 \mathrm{~m}$ orders which traverse the circle clockwise and counter-clockwise starting from every possible alternative. Note that the SPOC orders used in this proof are 'unbalanced', in that the most- and least-preferred alternatives are adjacent on the circle for every agent. Still, a similar dictatorship result can be proved even using orders that are 'Euclidean' on a circle, where preferences decrease uniformly in both directions from the peak (Schummer \& Vohra, 2002). It can also be shown that, with these Euclidean orders, the random dictatorship rule is group-strategyproof (Alon, Feldman, Procaccia, \& Tennenholtz, 2010b), and there is an intriguing randomised mechanism that is strategyproof and provides a $3 / 2$-approximation to the egalitarian social welfare (Alon, Feldman, Procaccia, \& Tennenholtz, 2010a).

Another interesting subdomain that we mentioned in the introduction is the combination of single-peaked and single-dipped (single-caved) orders with respect to the same underlying axis. This subdomain is particularly useful to model facility location, where the public facility may be perceived as a good or bad by different voters. Achuthankutty and Roy (2018) prove that the Gibbard-Satterthwaite Theorem holds for this subdomain. AlcaldeUnzu and Vorsatz (2018) also consider this kind of facility location problem, with the additional assumption that voters' pivotal points (their peak or dip) are public knowledge. For this model, they show that strategy-proof facility location mechanisms exist. When assuming Euclidean preferences that may be single-peaked or single-dipped, Feigenbaum and Sethuraman (2015) and Zou and Li (2015) also find strategy-proof mechanisms.

We conjecture that many other impossibility theorems from social choice theory will continue to apply after restricting to SPOC profiles. As an example, we will prove a celebrated result of Moulin (1988) in the SPOC context. He showed that every Condorcet-consistent voting rule fails participation. A voting rule $f$ is Condorcet-consistent if for every profile $P$ that admits a Condorcet winner $a$, we have $f(P)=a$. Participation states that no voter can strictly benefit by abstaining from an election. Formally, $f$ satisfies participation if for all profiles $P$, and all linear orders $\succ$, we have $f(P+\succ) \succcurlyeq f(P)$, where $P+\succ$ denotes the profile obtained from $P$ by adding a voter with preferences given by $\succ$. We will also write $P+k \cdot \succ$ for the profile obtained from $P$ by adding $k$ voters with preferences $\succ$.

Theorem 5 (No-Show Paradox for SPOC). For $m \geqslant 4$, there is no resolute voting rule on $S P O C$ profiles that is Condorcet-consistent and satisfies participation.

The proof is a straightforward adaptation of the proofs of Moulin (1988) and Brandt, Geist, and Peters (2017): most of the profiles in those proofs are SPOC. Somewhat more care needs to be taken while lifting the impossibility for $m=4$ to $m>4$ while maintaining the SPOC property.

Proof of Theorem 5. Let $m \geqslant 4$ and write $A=\left\{a_{1}, \ldots, a_{m-3}, b, c, d\right\}$. Consider the circle $C=a_{1} \triangleleft \cdots \triangleleft a_{m-3} \triangleleft b \triangleleft c \triangleleft d \triangleleft a_{1}$. In the following, for brevity, we write $\vec{a} b c d$ for the 
linear order $a_{1} \succ \cdots \succ a_{m-3} \succ b \succ c \succ d$. The following profiles are all SPOC on $C$ :

$$
\begin{array}{lrl}
P_{0} & = & 2 \cdot \vec{a} b c d+3 \cdot b c d \vec{a}+3 \cdot d \vec{a} b c+2 \cdot c d \vec{a} b \\
P_{1} & =2 \cdot \vec{a} b d c+2 \cdot \vec{a} b c d+3 \cdot b c d \vec{a}+3 \cdot d \vec{a} b c+2 \cdot c d \vec{a} b \\
P_{2} & = & 2 \cdot \vec{a} b c d+3 \cdot b c d \vec{a}+3 \cdot d \vec{a} b c+2 \cdot c d \vec{a} b+2 \cdot c d b \vec{a} \\
P_{3}=2 \cdot \vec{a} b d c+2 \cdot \vec{a} b c d+3 \cdot b c d \vec{a} & \\
P_{4}=2 \cdot \vec{a} b d c+2 \cdot \vec{a} b c d & 2 \cdot \vec{a} b c d+3 \cdot b c d \vec{a} & +d \vec{a} b c+2 \cdot c d \vec{a} b \\
P_{5}= & 3 \cdot d \vec{a} b c+2 \cdot c d \vec{a} b+2 \cdot c d b \vec{a} \\
P_{6}= &
\end{array}
$$

Let $f$ be a voting rule defined on SPOC profiles satisfying Condorcet-consistency and participation. By Condorcet-consistency, we have $f\left(P_{3}\right)=a_{1}, f\left(P_{4}\right)=d, f\left(P_{5}\right)=b$, and $f\left(P_{6}\right)=c$.

Write $A_{\vec{a}}=\left\{a_{1}, \ldots, a_{m-3}\right\}$. Consider $P_{0}$, and assume for now that $f\left(P_{0}\right) \in A_{\vec{a}} \cup\{b\}$. Then, since $f$ satisfies participation, also $f\left(P_{1}\right) \in A_{\vec{a}} \cup\{b\}$. By Condorcet-consistency, $f\left(P_{3}\right)=a_{1}$. Since $P_{1}$ can be obtained from $P_{3}$ by adding $d \vec{a} b c$ and $c d \vec{a} b$ voters, participation implies that $f\left(P_{1}\right) \in\left\{c, d, a_{1}\right\}$. Hence $f\left(P_{1}\right)=a_{1}$. But, by Condorcet-consistency, $f\left(P_{4}\right)=$ $d$; and since $P_{1}$ can be obtained from $P_{4}$ by adding $b c d \vec{a}$ voters, participation implies $f\left(P_{1}\right) \in\{b, c, d\}$, a contradiction.

Since we arrived at a contradiction, we must have $f\left(P_{0}\right) \notin A_{\vec{a}} \cup\{b\}$, and so $f\left(P_{0}\right) \in\{c, d\}$. Thus, by participation, also $f\left(P_{2}\right) \in\{c, d\}$. By Condorcet-consistency, $f\left(P_{5}\right)=b$, and thus by participation, $f\left(P_{2}\right) \in A_{\vec{a}} \cup\{d, b\}$, and thus $f\left(P_{2}\right)=d$. But by Condorcet-consistency, $f\left(P_{6}\right)=c$ and thus by participation, $f\left(P_{2}\right) \in A_{\vec{a}} \cup\{b, c\}$, a contradiction.

Since either case leads to a contradiction, there can be no voting rule $f$ with the desired properties.

As described in the next section, further impossibilities about tournament-based rules can be deduced from Lemma 6.

\section{Kemeny's and Young's Rules}

In this section, we will consider the problem of determining an election winner according to two well-known voting rules, Young's rule and Kemeny's rule, that are NP-hard to evaluate in general (Bartholdi III, Tovey, \& Trick, 1989; Rothe, Spakowski, \& Vogel, 2003; Hemaspaandra, Spakowski, \& Vogel, 2005). We will be interested to see whether these problems can be solved in polynomial time for SPOC profiles. We leave the complexity of Dodgson's rule for SPOC profiles for future work.

Kemeny's rule is a rank aggregation rule: Given a profile $P$ over $A$, its aim is to produce a consensus ranking over $A$. Suppose $r$ is a linear order over $A$. Its Kemeny score is

$$
\sum_{i \in N} \mid\left\{(x, y) \in A \times A: x \succ_{i} y \text { and } x \succ_{r} y\right\} \mid
$$

the number of pairwise agreements of $r$ with $P$. A Kemeny ranking is a linear order $r$ with maximum Kemeny score. (Equivalently, one can define a Kemeny ranking as a 
linear order that minimises the number of pairwise disagreements.) While it is NP-hard to find a Kemeny ranking (Bartholdi III et al., 1989), this problem is easy for singlepeaked profiles whose transitive majority relation is easily seen to give rise to a Kemeny ranking. For SPOC preferences, the situation is less clear: the Condorcet paradox profile $\left(x \succ_{1} y \succ_{1} z, y \succ_{2} z \succ_{2} x, z \succ_{3} x \succ_{3} y\right)$ on 3 alternatives is SPOC, so SPOC does not guarantee a transitive majority relation. In fact, as we now show, SPOC does not guarantee anything at all about the majority relation, which will imply that the SPOC restriction does not make the problem of computing Kemeny rankings easier.

If $P$ is a profile of linear orders, and $x, y \in A$, we write $n_{x, y}=\left|i \in N: x_{i} y\right|$ for the number of voters who prefer $x$ to $y$. The majority margin of $x$ over $y$ is $m_{x, y}=n_{x, y}-n_{y, x}$. Note that $m_{x, y}>0$ if and only if a strict majority of voters prefer $x$ to $y$. Note that $m_{x, y} \in \mathbb{Z}$ has the same parity as $|N|$ (since $m_{x, y}=n_{x, y}-\left(n-n_{x, y}\right)=2 n_{x, y}-n$ ), and so either all majority margins are even, or all are odd. The collection $\left(m_{x, y}\right)_{x, y \in A}$ is known as a weighted majority tournament. McGarvey's (1953) theorem (and its refinement by Debord, 1987) states that any such collection of integers, all of the same parity, is induced as the weighted majority tournament of some profile $P$ of linear orders. We can show that the same result holds when we additionally require that $P$ is SPOC.

Lemma 6 (McGarvey's theorem for SPOC). All (weighted) majority tournaments are inducible by SPOC profiles.

Proof. Fix a circle $C$ with $x_{1} \triangleleft x_{2} \triangleleft \cdots \triangleleft x_{m}$. For any $x_{i}, x_{j} \in A$ consider the profile $P_{x_{i}, x_{j}}$ consisting of the following two votes which are single-peaked on $C$, with subscripts taken modulo $m$ :

$$
\begin{aligned}
& x_{i+1} \succ \cdots \succ x_{j-1} \succ x_{i} \succ x_{j} \succ x_{j+1} \succ \cdots \succ x_{i-1} \\
& x_{i-1} \succ \cdots \succ x_{j+1} \succ x_{i} \succ x_{j} \succ x_{j-1} \succ \cdots \succ x_{i+1}
\end{aligned}
$$

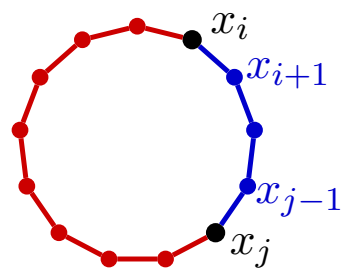

The profile $P_{x_{i}, x_{j}}$ induces a majority tournament with all margins 0 except that $m_{x_{i}, x_{j}}=$ $-m_{x_{j}, x_{i}}=2$.

Suppose we are given a collection $\left(m_{x, y}\right)_{x, y \in A}$ of even integers. Then consider the profile $P$ which contains, for each pair $x_{i}, x_{j}$ with $m_{x_{i}, x_{j}}>0$, exactly $m_{x_{i}, x_{j}} / 2$ copies of the profile $P_{x_{i}, x_{j}}$. Then $P$ induces $\left(m_{x, y}\right)_{x, y \in A}$, and $P$ is SPOC on $C$.

Suppose we are given a collection $\left(m_{x, y}\right)_{x, y \in A}$ of odd integers. Then consider the profile $P$ containing one voter with $x_{1} \succ \cdots \succ x_{m}$, and also for each pair $x_{i}, x_{j}$ with $i<j$, if $m_{x_{i}, x_{j}}>0$ then $\left(m_{x_{i}, x_{j}}-1\right) / 2$ copies of $P_{x_{i}, x_{j}}$, and if $m_{x_{i}, x_{j}}<0$ then $\left(m_{x_{i}, x_{j}}+1\right) / 2$ copies of $P_{x_{j}, x_{i}}$. Then $P$ induces $\left(m_{x, y}\right)_{x, y \in A}$, and $P$ is SPOC on $C$.

Now, the Kemeny score of a ranking $r$ as defined above is clearly equal to $\sum_{x, y \in A: x \succ_{r} y} n_{x, y}$. Since $n_{x, y}=\left(m_{x, y}+n\right) / 2$, we see that the Kemeny score is a function of the weighted majority tournament of a profile. Since the profiles in the proof of McGarvey's theorem above can be produced in polynomial time, the hardness of Kemeny in the general case carries over.

Theorem 7. Given a SPOC profile of linear orders and a number $k$, it is NP-complete to decide whether there exists a linear order with Kemeny score at least $k$. 
Proof. Containment in NP is clear. We reduce from the unconstrained problem of deciding whether in a given profile $P$, there exists a linear order with Kemeny score at least $k$, which is known to be NP-complete (Bartholdi III et al., 1989). Given such an instance, use the construction of Lemma 6 to produce a SPOC profile $P^{\prime}$ such that the majority margins for any pair $x, y$ of alternatives are the same in $P$ and $P^{\prime}$. Write $n$ for the number of voters in $P$, and $n^{\prime}$ for the number of voters in $P^{\prime}$. Let $r$ be some linear order. Its Kemeny score in $P^{\prime}$ is

$$
\sum_{x, y \in A: x \succ_{r} y} n_{x, y}^{P^{\prime}}=\sum_{x, y \in A: x \succ_{r} y}\left(m_{x, y}+n^{\prime}\right) / 2=\sum_{x, y \in A: x \succ_{r} y} n_{x, y}^{P}+\left(\begin{array}{c}
m \\
2
\end{array}\right)\left(n^{\prime}-n\right) / 2 .
$$

Hence, there exists a linear order with Kemeny score $r+\left(\begin{array}{c}m \\ 2\end{array}\right)\left(n^{\prime}-n\right) / 2$ in $P^{\prime}$ if and only there exists a linear order with Kemeny score $r$ in $P$.

For the same reason, essentially all negative (axiomatic or computational) results about voting rules based on (weighted) tournaments (see Brandt, Brill, \& Harrenstein, 2016; Fischer, Hudry, \& Niedermeier, 2016) still hold restricted to SPOC preferences.

Young's rule. Given a profile $P$ over $A$, an alternative $c \in A$ is a Condorcet winner if for every $b \in A \backslash\{c\}$, a majority of voters in $P$ strictly prefers $c$ to $b$. The Young score of an alternative $c \in A$ is the minimum number of voters that have to be deleted from $P$ so that $c$ becomes a Condorcet winner. Then, Young's rule selects all alternatives with minimum Young score as winners. It is known that Young winners can be found in polynomial time for single-peaked preferences (Brandt et al., 2015), since in this case Condorcet winners always exists when the number of voters $n$ is odd; and the case with $n$ even is also handled easily.

Because SPOC does not guarantee the existence of a Condorcet winner, a different approach is needed. We will use the interpretation of SPOC in terms of intervals of the underlying circle to give a polynomial-time algorithm that calculates the Young score of every alternative; clearly this algorithm can then be run repeatedly to find a Young winner. Of course, our algorithm also works for preferences single-peaked on a line; while the algorithm of Brandt et al. (2015) returns only a Young winner, our algorithm can find the Young score of any given alternative. ${ }^{2}$ Note that precise definitions of Young scores differ slightly: sometimes it is only required that an alternative be made a weak Condorcet winner through voter deletion; our algorithm can be easily adapted for this alternative definition.

Theorem 8. For SPOC profiles, the Young score of an alternative can be computed in $O\left(m n^{2}\right)$ time.

Proof. We fix an axis $\triangleleft \in C$ that starts with the alternative $a$ whose Young score we want to compute; let $a \triangleleft b \triangleleft \cdots \triangleleft c$ ( $b$ is the candidate right of $a, c$ is the rightmost candidate). We partition voters into two sets: $N_{1}=\left\{i \in N: b \succ_{i} a\right\}$ and $N_{2}=N \backslash N_{1}$. Since $P$ is SPOC, for any voter $i$, the set $I_{i}:=\left\{d \in A: d \succ_{i} a\right\}$ forms an interval of $\triangleleft$. Voters in $N_{1}$ correspond to intervals containing $b$; voters in $N_{2}$ correspond to intervals containing $c$ but not $b$, and to empty intervals. Figure 2 illustrates the situation: For each voter $i$, an arc indicates the set $I_{i}$. The red arcs on the right belong to voters from $N_{1}$, and the blue $\operatorname{arcs}$ on the left belong to voters from $N_{2}$.

2. Fitzsimmons and Hemaspaandra (2020, Theorem 10) give an algorithm for calculating Dodgson scores in single-peaked profiles. 


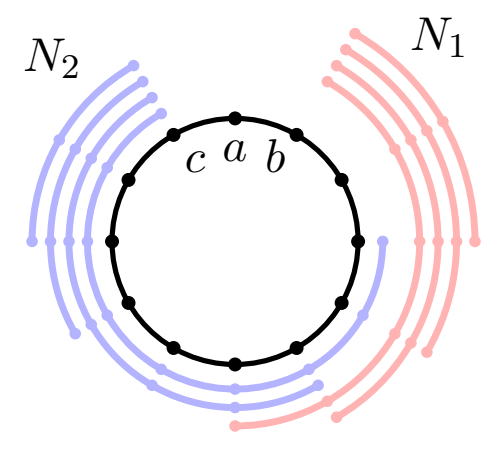

Figure 2: Illlustration of the proof of Theorem 8, for a profile with eight voters. For each voter, an arc indicates the set of alternatives preferred to $a$.

The idea behind our algorithm is that if there are voters $i$ and $j$ with $I_{i} \subseteq I_{j}$, then it is at least as profitable (for purposes of making $a$ the Condorcet winner) to remove voter $j$ as to remove voter $i$. Now note that the intervals $I_{i}$ of voters in $N_{1}$ are nested by set inclusion, and similarly for voters in $N_{2}$. Thus, we let $N_{1}^{-r}$ and $N_{2}^{-s}$ denote the subsets of $N_{1}$ and $N_{2}$ obtained by deleting, respectively, the $r$ and $s$ voters from $N_{1}$ and $N_{2}$ that have the $r$ and $s$ largest (with respect to set inclusion) intervals $I_{i}$. Because of the nesting property, if there is a way of deleting $r$ and $s$ voters from $N_{1}$ and $N_{2}$ that makes $a$ the Condorcet winner, then the deletions giving $N_{1}^{-r}$ and $N_{2}^{-s}$ also make $a$ the Condorcet winner.

These observations suggest the following simple algorithm: For every pair $(r, s)$ with $0 \leqslant r \leqslant\left|N_{1}\right|$ and $0 \leqslant s \leqslant\left|N_{2}\right|$, we check whether $a$ is the Condorcet winner in $N_{1}^{-r} \cup N_{2}^{-s}$. We return a pair $\left(r^{*}, s^{*}\right)$ with $r^{*}+s^{*}$ minimum for which this is the case. Then the Young score of $a$ is $r^{*}+s^{*}$. If no such pair exists, the Young score of $a$ is infinite.

To see that this algorithm can be run in $O\left(m n^{2}\right)$ time, we show how to check in $O(m)$ time whether $a$ is the Condorcet winner in $N_{1}^{-r} \cup N_{2}^{-s}$. To do so, we precompute for every $x \in A \backslash\{c\}, 0 \leqslant r \leqslant\left|N_{1}\right|$, and $0 \leqslant s \leqslant\left|N_{2}\right|$ the numbers

$$
\begin{aligned}
& d_{r}^{1}(x)=\left|\left\{i \in N_{1}^{-r}: a \succ_{i} x\right\}\right|-\left|\left\{i \in N_{1}^{-r}: x \succ_{i} a\right\}\right|, \\
& d_{s}^{2}(x)=\left|\left\{i \in N_{2}^{-s}: a \succ_{i} x\right\}\right|-\left|\left\{i \in N_{2}^{-s}: x \succ_{i} a\right\}\right| .
\end{aligned}
$$

Note that $a$ is a Condorcet winner in $N_{1}^{-r} \cup N_{2}^{-s}$ if and only if for all $x \in A \backslash\{c\}$ it holds that $d_{r}^{1}(x)+d_{s}^{2}(x)>0$. The quantities $d_{r}^{1}(x)$ and $d_{s}^{2}(x)$ can be precomputed in $O\left(m n^{2}\right)$ time. Verifying whether $d_{r}^{1}(x)+d_{s}^{2}(x)>0$ requires constant time and hence $O(m)$ time for every $x \in A \backslash\{c\}$.

\section{Multi-Winner Elections}

Much recent work has studied voting rules that select not a single winner, but a committee $W \subseteq A$ of candidates, where $|W|=k$ has some desired size $k$ (see, e.g., a recent survey by Faliszewski, Skowron, Slinko, \& Talmon, 2017). Depending on the context, we may wish this committee to have different properties. For example, we may aim for a representative committee in which as many voters as possible have a good representative, or we may aim 
for a proportional committee in which subgroups of the voters are represented by committee members in proportion to the subgroup size. Many of the commonly-studied multi-winner rules optimise an objective function over the set of all committees. Unsurprisingly, many of them are NP-hard to evaluate. In this section, we show that several popular rules can be evaluated in polynomial time when preferences are single-peaked on a circle.

Chamberlin and Courant (1983) introduced a rule that aims for a committee that represents as many voters as well as possible. It is usually defined for profiles of linear orders. According to this rule, each voter $i$ is represented by $i$ 's favourite (highest-ranked) alternative in $W$; suppose this is $c_{i} \in W$. Then, we take the 'utility' of voter $i$ to be the Borda score (i.e., position counting from the bottom) of $c_{i}$ in $i$ 's ranking. The Chamberlin-Courant rule selects a committee of size $k$ that maximises the sum of voter utilities. By replacing Borda scores by other scoring vectors, we obtain a whole family of rules. The class of OWA-based rules, as defined below, is a further generalization of this idea.

Finding a winning committee under the Chamberlin-Courant rule is known to be NPhard for Borda scores (Lu \& Boutilier, 2011). Betzler et al. (2013) showed that this problem becomes easy when the input profile is single-peaked. Their algorithm starts by running a recognition algorithm for single-peakedness on the input to obtain an underlying axis $\triangleleft$ on which the profile is single-peaked. Then, they run a dynamic programming algorithm which constructs an optimal committee. Roughly, this dynamic program successively considers left prefixes of the axis $\triangleleft$, and constructs an optimal committee using only candidates from the prefix. Unfortunately, it is unclear how to extend this approach to preferences singlepeaked on a circle, since a circle does not have a left endpoint where we could start the dynamic program.

Thus, we follow a different approach: We design an integer linear programming (ILP) formulation encoding the winner determination problem. We then show that the matrix of coefficients appearing in the constraints of this ILP is totally unimodular whenever the input profile is SPOC. A well-known result states that ILPs with totally unimodular constraint matrices are optimally solved by their LP relaxations (Hoffman \& Kruskal, 1956), and can thus be solved in polynomial time.

As a preview, for the Chamberlin-Courant rule based on Borda scores, our general ILP formulation looks as follows:

$$
\begin{array}{rlrl}
\text { maximise } & \sum_{i \in N} \sum_{r \in[m]} x_{i, r} & \\
\text { subject to } & \sum_{c \in C} y_{c}=k & \\
x_{i, r} & \leqslant \sum_{c: \operatorname{rank}(c) \leqslant r} y_{c} & & \text { for } i \in N, r \in[m] \\
x_{i, r} & \in\{0,1\} & \text { for } i \in N, r \in[m] \\
y_{c} & \in\{0,1\} & \text { for } c \in C
\end{array}
$$

This formulation has one binary variable $y_{c}$ for each candidate $c \in C$, indicating whether candidate $c$ is part of the committee. Constraint (2) requires that the committee contains 
exactly $k$ candidates. The binary variables $x_{i, r}$ indicate whether voter $i \in N$ ranks some committee member in rank $r$ or better; this interpretation is implemented by the constraints (3) together with the fact that the program is maximising the sum of all $x_{i, r}$. Note that if the best candidate for voter $i \in N$ in the committee described by the variables $\left(y_{c}\right)_{c \in C}$ is in rank 4 , say, then the program will set $x_{i, 1}=\cdots=x_{i, 3}=0$ and $x_{i, 4}=\cdots=x_{i, m}=1$. Thus, the contribution of voter $i$ to the objective function is $m-4+1$, which is the Borda score (plus one) of $i$ 's representative. Thus, the objective function of this program correctly encodes Chamberlin-Courant.

Our approach works not only for the Chamberlin-Courant rule, but for a large class of rules introduced by Skowron, Faliszewski, and Lang (2016), called OWA-based rules (OWA stands for ordered weighted average). An example of such a rule is Chamberlin-Courant, but defined so that voter $i$ 's utility is the sum of the Borda scores of $i$ 's best two members of the committee. Let us formally describe this class of rules. We will give a definition that works for weak order inputs, and so this class includes rules that work for linear order profiles, and for approval profiles. Given a preference profile, as a first step the rule converts preferences into numerical scores, using a positional scoring system. Suppose that $\succcurlyeq$ is a weak order over $A$. Then we can uniquely partition $A=A_{1} \cup \cdots \cup A_{q}$ into disjoint non-empty sets such that $A_{1} \succ \cdots \succ A_{q}$ and such that $a \sim b$ for all $a, b \in A_{r}$ for $r \in[q]$. The sets $A_{r}$ are called the indifference classes of the weak order $\succcurlyeq$. Now, for an alternative $a \in A$, if $a \in A_{r}$, the rank of $a$ in $\succcurlyeq$ is $r$, and we write $\operatorname{rank}_{\succcurlyeq}(a)=r$. Thus, the alternatives with rank 1 are the most-preferred alternatives. If we are given a profile $P$, then we write $\operatorname{rank}_{i}(a)$ for $i \in N$ and $a \in A$ for the rank of $a$ in voter $i$ 's preferences. A score vector is a vector $\mathbf{s} \in \mathbb{R}^{m}$ such that $s_{1} \geqslant s_{2} \geqslant \cdots \geqslant s_{m}$. Common examples are $\mathbf{s}=(m-1, m-2, \ldots, 0)$ for Borda scores and $\mathbf{s}=(1,0, \ldots, 0)$ for plurality scores. Given such a score vector $\mathbf{s}$, we say that voter $i \in N$ assigns the score $s_{\operatorname{rank}_{i}(a)}$ to alternative $a \in A$, and we write $\mathbf{s}(i, a)=s_{\operatorname{rank}_{i}(a)}$. This is the standard definition when preferences are given by linear orders. ${ }^{3}$ If a voter submits an approval ballot, and we use plurality scores, then the voter assigns score 1 to all approved alternatives and score 0 to the remaining alternatives. Note that whenever $a \succcurlyeq_{i} b$ then $\mathbf{s}(i, a) \geqslant \mathbf{s}(i, b)$.

The utility a voter derives from a committee under an OWA-based rule will be a linear combination of the scores assigned to the candidates in the committee, and these values are calculated using an OWA operator. A weight vector $\boldsymbol{\alpha} \in \mathbb{R}^{k}$ defines an ordered weighted average (OWA) operator as follows: Given any vector $\mathbf{x} \in \mathbb{R}^{k}$, first sort the entries of $\mathbf{x}$ into non-increasing order, so that $x_{\sigma(1)} \geqslant \ldots \geqslant x_{\sigma(k)}$; second, apply the weights: the ordered weighted average of $\mathbf{x}$ with weights $\boldsymbol{\alpha}$ is given by $\boldsymbol{\alpha}(\mathbf{x}):=\sum_{i=1}^{k} \alpha_{i} x_{\sigma(i)}$. For example, if $\boldsymbol{\alpha}=$ $(1,0, \ldots, 0)$, then $\boldsymbol{\alpha}(\mathbf{x})=x_{\sigma(1)}=\max _{i \in[k]} x_{i}$, so that this operator returns the maximum of the vector x. Alternatively, if $\boldsymbol{\alpha}=(1,1, \ldots, 1)$, then $\boldsymbol{\alpha}(\mathbf{x})=\sum_{i=1}^{k} x_{\sigma(i)}=\sum_{i=1}^{k} x_{i}$, so that this operator gives the sum of the numbers in $\mathbf{x}$.

3. Often, it will be unnatural to use the same scoring system both for linear orders and other weak orders (such as approval ballots). For example, one might adjust the definition of Borda scores when weak orders are submitted (Chebotarev \& Shamis, 1998). It is straightforward to adjust our algorithm to allow for different scoring systems for different order types, or even for different scoring systems for each agent; all that's needed is to adjust the coefficients in the objective function of the ILP. Often, it will also be natural to restrict the input of the rule to just linear orders, or to just approval ballots. We have chosen the current formulation for ease of presentation. 
Given a profile $P$, a scoring vector $\mathbf{s}$, and an OWA operator $\boldsymbol{\alpha}$, we define the utility of a committee $W=\left\{c_{1}, \ldots, c_{k}\right\}$ as

$$
U(\mathbf{s}, \boldsymbol{\alpha}, W)=\sum_{i \in N} \boldsymbol{\alpha}\left(\mathbf{s}\left(i, c_{1}\right), \ldots, \mathbf{s}\left(i, c_{k}\right)\right)
$$

Then the OWA-based multi-winner rule based on $\mathbf{s}$ and $\boldsymbol{\alpha}$ outputs a committee $W$ of size $k$ for which $U(\mathbf{s}, \boldsymbol{\alpha}, W)$ is maximum.

For example, choosing $\boldsymbol{\alpha}=(1,0, \ldots, 0)$ and $\mathbf{s}=(m-1, m-2, \ldots, 0)$ (Borda scores) gives us the Chamberlin-Courant rule, where each voter derives as utility the score of their favourite committee member. Choosing $\boldsymbol{\alpha}=(1,1,0, \ldots, 0)$ gives us the analogue of Chamberlin-Courant mentioned above, where voters obtain as utility the sum of the scores of their favourite two members of the committee (this rule is sometimes known as 2-Borda (Faliszewski, Skowron, Slinko, \& Talmon, 2017)).

When we restrict our attention on dichotomous weak orders, then the OWA-based rule applied to profiles with approval votes with $\boldsymbol{\alpha}=\left(1, \frac{1}{2}, \ldots, \frac{1}{k}\right)$ and scores $\mathbf{s}=(1,0)$ gives us Proportional Approval Voting (PAV), a voting rule that has received much attention in the recent literature (Aziz, Brill, Conitzer, Elkind, Freeman, \& Walsh, 2017; Janson, 2016; Lackner \& Skowron, 2018). Defining it more explicitly, PAV returns the committee $W \subseteq C$ with $|W|=k$ maximising $\sum_{i \in N} 1+\frac{1}{2}+\cdots+\frac{1}{\left|A_{i} \cap W\right|}$, where $A_{i}$ is the set of candidates that voter $i$ approves. For the special case of PAV, the ILP formulation we will give for general OWA-based rules looks as follows:

$$
\begin{array}{rlrl}
\text { maximise } & \sum_{i \in N} \sum_{\ell \in[k]} \frac{1}{\ell} \cdot x_{i, \ell} & \\
\text { subject to } \sum_{c \in C} y_{c}=k & \\
\sum_{\ell \in[k]} x_{i, \ell} & \leqslant \sum_{c \in A_{i}} y_{c} & & \text { for } i \in N \\
x_{i, \ell} & \in\{0,1\} & \text { for } i \in N, \ell \in[k] \\
y_{c} & \in\{0,1\} & \text { for } c \in C
\end{array}
$$

Again, this formulation has one binary variable $y_{c}$ for each candidate $c \in C$. The binary variables $x_{i, \ell}$ indicate whether voter $i \in N$ approves of at least $\ell$ candidates in the committee; this interpretation is implemented by the constraints (3) together with the fact that the coefficients of $x_{i, \ell}$ in the objective function decrease with $\ell$. Our most general ILP formulation will merge the ideas behind (PAV-ILP) and (CC-ILP).

Our polynomial-time result will only work for non-increasing OWA vectors $\boldsymbol{\alpha}$ with $\alpha_{1} \geqslant \cdots \geqslant \alpha_{k}$. For example, this excludes the rule where voters are represented by their least-favourite committee member, or by their median committee member. While such rules may be sensible in some settings (Skowron, 2015), this restriction seems mild for most contexts.

Next, let us give an overview about total unimodularity. A matrix $A=\left(a_{i j}\right)_{i j} \in \mathbb{Z}^{m \times n}$ with $a_{i j} \in\{-1,0,1\}$ is called totally unimodular if every square submatrix $B$ of $A$ has 
$\operatorname{det} B \in\{-1,0,1\}$. (The rows and columns of $B$ need not occur contiguously in $A$.) The following results are well-known. Proofs and much more about their theory can be found in the textbook by Schrijver (1998).

Theorem 9 (see Schrijver, 1998, Theorem 19.1). Suppose $A \in \mathbb{Z}^{m \times n}$ is a totally unimodular matrix, $b \in \mathbb{Z}^{m}$ is an integral vector of right-hand sides, and $c \in \mathbb{Q}^{n}$ is an objective vector. Then the linear program

$$
\max c^{T} x \text { subject to } A x \leqslant b
$$

has an integral optimum solution, which is a vertex of the polyhedron $\{x: A x \leqslant b\}$. Thus, the integer linear program

$$
\max c^{T} x \text { subject to } A x \leqslant b, x \in \mathbb{Z}^{n}
$$

is solved optimally by its linear programming relaxation (LP).

An optimum solution to (ILP) can be found in polynomial time (Maurras, Truemper, \& Akgül, 1981; Tardos, 1986). We will now state some elementary results about totally unimodular matrices, which allows us to build new matrices from old.

Proposition 10 (see Schrijver, 1998, chapter 19). If $A$ is totally unimodular, then so is

1. its transpose $A^{T}$,

2. the matrix $[A \mid-A]$ obtained from $A$ by appending the negated columns of $A$,

3. the matrix $[A \mid I]$ where $I$ is the identity matrix,

4. any matrix obtained from A through permuting or deleting rows or columns.

In particular, from (3) and (4) it follows that appending a unit column $(0, \ldots, 1, \ldots, 0)^{T}$ will not destroy total unimodularity. Further, using these transformations, we can see that Theorem 9 remains true even if we add to $(\mathrm{P})$ constraints giving lower and upper bounds to some variables, if we replace some of the inequality constraints by equality constraints, or change the direction of an inequality.

Recall that a binary matrix $A=\left(a_{i j}\right) \in\{0,1\}^{m \times n}$ has the consecutive ones property if its columns can be permuted such that the 1-entries of each row form an interval. The key result that will allow us to connect single-peaked preferences to total unimodularity is as follows:

Proposition 11 (see Schrijver, 1998, page 279). Every binary matrix with the consecutive ones property is totally unimodular.

We remark that by a celebrated result of Seymour (1980), it is possible to decide in polynomial time whether a given matrix is totally unimodular, though we do not use this fact.

We are now ready to prove our main result, that OWA-based rules are easy to compute for SPOC profiles. 
Theorem 12. Given a SPOC profile $P$, and an $O W A$-based rule specified by a scoring vector $\mathbf{s}$ and a non-increasing OWA operator $\boldsymbol{\alpha}$, a winning committee can be found in polynomial time.

Proof. We begin by showing the result for single-peaked profiles, and later show how to modify the argument for SPOC profiles.

Let $P$ be a preference profile, and let $k$ be the target committee size. Consider the following integer linear program, whose optimal solutions correspond to winning committees under the OWA-based rule with operator $\boldsymbol{\alpha}$ and scoring vector s. In the program, we write $s_{m}^{\prime}=s_{m}$, and for each $r=1, \ldots, m-1$, we write $s_{r}^{\prime}=s_{r}-s_{r+1} \geqslant 0$. Thus, for every $r \in[m]$, we have that $s_{r}=\sum_{p=r}^{m} s_{p}^{\prime}$.

$$
\begin{aligned}
& \text { maximise } \sum_{i \in N} \sum_{\ell \in[k]} \sum_{r \in[m]} \alpha_{\ell} \cdot s_{r}^{\prime} \cdot x_{i, \ell, r} \\
& \text { subject to } \sum_{c \in A} y_{c}=k \\
& \sum_{\ell \in[k]} x_{i, \ell, r} \leqslant \sum_{c: \operatorname{rank}_{i}(c) \leqslant r} y_{c} \quad \text { for } i \in N, r \in[m] \\
& x_{i, \ell, r} \in\{0,1\} \quad \text { for } i \in N, \ell \in[k], r \in[m] \\
& y_{c} \quad \in\{0,1\} \quad \text { for } c \in A
\end{aligned}
$$

Every feasible solution $\left(\left(x_{i, \ell, r}\right)_{i, \ell, r},\left(y_{c}\right)_{c}\right)$ to the ILP corresponds to a committee $W=$ $\left\{c \in A: y_{c}=1\right\}$. Due to the constraint $\sum_{c \in A} y_{c}=k$, we have that $|W|=k$, so this is a committee of the required size. Next suppose that $S=\left(\left(x_{i, \ell, r}\right)_{i, \ell, r},\left(y_{c}\right)_{c}\right)$ is an optimal solution to the ILP. We may assume that under $S$, all constraints (3) are satisfied with equality, since otherwise we could set additional variables $x_{i, \ell, r}$ to 1 without affecting feasibility, and without lowering the objective value of the solution (because the coefficient $\alpha_{\ell} \cdot s_{r}^{\prime}$ of $x_{i, \ell, r}$ is non-negative). Further, this operation does not change the committee $W$. Now fix a voter $i \in N$ and a rank $r \in[m]$. Suppose that there are $L$ candidates in $W$ which voter $i$ places in rank $r$ or better, i.e.,

$$
L=\left|W \cap\left\{c \in A: \operatorname{rank}_{i}(c) \leqslant r\right\}\right| .
$$

By our assumption that the constraint (3) is satisfied in $S$ with equality, exactly $L$ of the variables $x_{i, \ell, r}$ for $\ell \in[k]$ are set to 1 in $S$. By our assumption that the vector $\boldsymbol{\alpha}$ is nonincreasing, the coefficients $\alpha_{\ell} \cdot s_{r}^{\prime}$ of $x_{i, \ell, r}$ in the objective function are non-increasing as $\ell$ goes from 1 to $k$. Hence, we may assume without loss of generality that in $S$, we have

$$
x_{i, 1, r}=\cdots=x_{i, L, r}=1 \quad \text { and } \quad x_{i, L+1, r}=\cdots x_{i, k, r}=0 .
$$

Then it follows that for $i \in N, \ell \in[k], r \in[m]$, we have

$$
x_{i, \ell, r}=1 \quad \text { if and only if } W \text { contains at least } \ell \text { candidates } c \text { with } \operatorname{rank}_{i}(c) \leqslant r .
$$

Fix $i \in N$ and $\ell \in[k]$. Write $W=\left\{c_{1}, \ldots, c_{k}\right\}$ so that $c_{1} \succcurlyeq_{i} \cdots \succcurlyeq_{i} c_{k}$. Then it follows that, for every $r \in[m]$,

$$
x_{i, \ell, r}=1 \text { if and only if } \operatorname{rank}_{i}\left(c_{\ell}\right) \leqslant r .
$$


(If $x_{i, \ell, r}=1$, then $W$ contains at least $\ell$ candidates $c$ with $\operatorname{rank}_{i}(c) \leqslant r$, and so in particular $c_{1}, \ldots, c_{\ell}$ must have rank $r$ or better. Conversely, if $\operatorname{rank}_{i}\left(c_{\ell}\right) \leqslant r$ then $c_{1}, \ldots, c_{\ell}$ all have rank $r$ or better, so there are at least $\ell$ candidates in $W$ with rank $r$ or better, and so $x_{i, \ell, r}=1$.) Hence, the utility of voter $i$ in committee $W$ is

$$
\begin{aligned}
\boldsymbol{\alpha}\left(s_{\operatorname{rank}_{i}\left(c_{1}\right)}, \ldots, s_{\operatorname{rank}_{i}\left(c_{k}\right)}\right) & =\sum_{\ell \in[k]} \alpha_{\ell} \cdot s_{\operatorname{rank}_{i}\left(c_{\ell}\right)} \\
& =\sum_{\ell \in[k]} \alpha_{\ell} \cdot\left(\sum_{r=\operatorname{rank}_{i}\left(c_{\ell}\right)}^{m} s_{r}^{\prime}\right) \\
& =\sum_{\ell \in[k]} \sum_{r \in[m]} \alpha_{\ell} \cdot s_{r}^{\prime} \cdot x_{i, \ell, r} .
\end{aligned}
$$

Summing over all $i \in N$, we see that the objective value of solution $S$ to the ILP equals $U(\mathbf{s}, \boldsymbol{\alpha}, W)$, the total utility of the committee $W$ under the OWA-based rule. Thus, the ILP correctly encodes the winner determination problem.

Next suppose that the profile $P$ is single-peaked. Consider the matrix $M$ of coefficients in the constraints of the ILP. Let $M^{\prime}$ be the submatrix consisting only of the columns corresponding to the variables $\left(y_{c}\right)_{c \in A}$. Then $M^{\prime}$ has one row consisting of only 1s (corresponding to constraint (2)), and for each $i \in N$ and $r \in[m]$ a row whose 1-entries encode the set $\left\{c \in A: \operatorname{rank}_{i}(c) \leqslant r\right\}$. Note that each of these sets is a top-initial segment of the preference order of $i$, and hence (see Section 2) an interval of the axis on which $P$ is single-peaked. Therefore $M^{\prime}$ is a consecutive ones matrix (with the columns ordered according to the axis). Thus $M^{\prime}$ is totally unimodular by Proposition 11. Now, the matrix $M$ is obtained from $M^{\prime}$ by appending columns corresponding to the variables $x_{i, \ell, r}$. Each of these variables occurs in only 1 constraint of type (3) with coefficient \pm 1 (the sign depends on how we rearrange constraint (3) to bring all variables to one side). Thus, the column of the variable $x_{i, \ell, r}$ is a (negative) unit column, and so by Proposition 10, the matrix remains totally unimodular after appending it. Thus, $M$ is totally unimodular. (Technically, we also need to include the constraints $0 \leqslant x_{i, \ell, r} \leqslant 1$ and $0 \leqslant y_{c} \leqslant 1$, but these are unit rows which can again be added without destroying total unimodularity.) Thus, by Theorem 9, the ILP can be solved in polynomial time.

The above argument for total unimodularity does not go through if $P$ is SPOC but not single-peaked, because then the matrix $M^{\prime}$ only has the circular ones property. However, we can rearrange the ILP in such a way that we can show total unimodularity. This is a standard technique described in a useful survey by Dom (2009, Sec 4.1.4). Before we begin, let us note the following general fact: suppose we are given a system of constraints

$$
f(\mathbf{x})=0 \text { and } g_{j}(\mathbf{x}) \leqslant 0 \text { for } j=1, \ldots, J .
$$

If in this system we replace one or more of the constraints $g_{j}(\mathbf{x}) \leqslant 0$ by $g_{j}(\mathbf{x})-f(\mathbf{x}) \leqslant 0$, then the set of feasible solutions $\mathbf{x}$ to the system does not change.

Let $P$ be a SPOC preference profile. Using the algorithms from Section 3, find a circle $C$ such that $P$ is single-peaked on $C$, and take some $\triangleleft \in C$ arbitrarily. For $i \in N$ and $r \in[m]$, write $T_{i, r}=\left\{c \in A: \operatorname{rank}_{i}(c) \leqslant r\right\}$. Then $T_{i, r}$ is a top-initial segment of $i$ 's vote. Since $P$ is single-peaked on $C, T_{i, r}$ is an interval of $C$. Thus, either $T_{i, r}$ or $A \backslash T_{i, r}$ is an 
interval of $\triangleleft$. Define the sets

$$
\begin{aligned}
& \Gamma_{1}=\left\{(i, r): i \in N, r \in[m] \text { such that } T_{i, r} \text { is an interval of } \triangleleft\right\}, \\
& \Gamma_{2}=\left\{(i, r): i \in N, r \in[m] \text { such that } A \backslash T_{i, r} \text { is an interval of } \triangleleft\right\} \backslash \Gamma_{1} .
\end{aligned}
$$

Then $\Gamma_{1}$ and $\Gamma_{2}$ form a partition of $N \times[m]$. Now consider the following integer linear program:

$$
\begin{aligned}
& \text { maximise } \sum_{i \in N} \sum_{\ell \in[k]} \sum_{r \in[m]} \alpha_{\ell} \cdot s_{r}^{\prime} \cdot x_{i, \ell, r} \\
& \text { subject to } \sum_{c \in A} y_{c}-k=0 \\
& \sum_{\ell \in[k]} x_{i, \ell, r} \leqslant \sum_{c: \operatorname{rank}_{i}(c) \leqslant r} y_{c} \quad \text { for } i \in N, r \in[m] \text { with }(i, r) \in \Gamma_{1} \\
& \sum_{\ell \in[k]} x_{i, \ell, r} \leqslant-\sum_{c: \operatorname{rank}_{i}(c)>r} y_{c}+k \quad \text { for } i \in N, r \in[m] \text { with }(i, r) \in \Gamma_{2} \\
& x_{i, \ell, r} \in\{0,1\} \quad \text { for } i \in N, \ell \in[k], r \in[m] \\
& y_{c} \in\{0,1\} \quad \text { for } c \in A
\end{aligned}
$$

The program (OWA-ILP-SPOC) is very similar to the original program (OWA-ILP). Note that constraint (2') is the same as (2) after rearranging. The constraints (3') are a selection of the constraints (3). Finally, constraints (3") are obtained from constraints (3) after subtracting constraint $\left(2^{\prime}\right)$. Since $\left(2^{\prime}\right)$ is an equality constraint, by the earlier mentioned general fact, we see that (OWA-ILP-SPOC) and (OWA-ILP) have the same set of feasible solutions. They also have the same objective function, and therefore (OWA-ILP-SPOC) also correctly encodes the problem of finding a winning committee.

Finally, we can prove that (OWA-ILP-SPOC) is totally unimodular, establishing the result that a winning committee can be found in polynomial time for SPOC profiles. Again take the constraint matrix $M$ and consider the submatrix $M^{\prime}$ corresponding to the variables $\left(y_{c}\right)_{c \in A}$. If we rearrange the columns of $M^{\prime}$ according to $\triangleleft$, then each row of $M^{\prime}$ consists of either of an interval of +1 s surrounded by $0 \mathrm{~s}$, or of an interval of $-1 \mathrm{~s}$ surrounded by 0 s (the latter arising from constraints (3")). Combining Propositions 11 and 10, we see that $M^{\prime}$ is totally unimodular. As before, $M$ is obtained from $M^{\prime}$ by adding columns with a single non-zero entry, so $M$ is also totally unimodular.

We obtain immediately the following two corollaries:

Corollary 13. For SPOC profiles, Chamberlin-Courant can be computed in polynomial time.

Corollary 14. For SPOC profiles, PAV can be computed in polynomial time.

Corollaries 13 and 14 clearly also apply to single-peaked profiles. For ChamberlinCourant, a corresponding results has already been established using dynamic programming 
(Betzler et al., 2013), but to the best of our knowledge this is the first polynomial-time algorithm for PAV on single-peaked profiles. ${ }^{4}$

An interesting question is whether the method of Theorem 12 can be further generalised. A possible generalization would be towards participatory budgeting, by introducing costs for candidates and replacing the committee size $k$ by a budget limit. Does winner determination of OWA-based rules remain easy on single-peaked or SPOC profiles in this setting? Since totally unimodular matrices can only include coefficients from $\{-1,0,1\}$, it seems unlikely that the packing constraint for non-unit costs can be implemented in this approach. However, we are not aware of a hardness result for this problem. It would also be interesting to see whether rules not part of the OWA-based family can be computed efficiently with single-peaked or SPOC profiles. Fluschnik, Skowron, Triphaus, and Wilker (2019) study the smoothed Nash product rule (which behaves similar to PAV but allows for non-binary utilities), and show that it is hard to compute even for single-peaked utilities.

The ILP formulation (OWA-ILP) from the proof of Theorem 12 is of independent interest for computing OWA-based rules; for example, it seems to have proven useful in the empirical study of Faliszewski, Szufa, and Talmon (2018). Indeed, the "algorithm" that we propose for computing OWA-based rules (i.e., solving the program (OWA-ILP) using an ILP solver) is correct for general preferences, and comes with a polynomial-time guarantee in case the algorithm's input is single-peaked. This is in contrast to other winner determination algorithms that exploit preference structure: most such algorithms are specialised, and do not work at all if their input fails to be appropriately structured.

\section{Discussion and Open Problems}

Our results show that restricted preference domains that behave unfavorably in terms of axiomatic properties might still be very useful for algorithmic purposes. Indeed, our algorithms for Young's rule and OWA-based committee selection rules demonstrate that it is possible to move to a larger class than single-peaked preferences while maintaining polynomial-time runtime bounds. ${ }^{5}$ Thus, our findings can be seen as a challenge to established algorithmic results based on restricted preferences: to which degree can their application domain be extended without resorting to super-polynomial algorithms? One open problem of this type asks whether Dodgson's rule can be evaluated in polynomial time for SPOC profiles.

Our definition of SPOC is not the only sensible definition. One alternative definition that would fit into the generalised notion of single-peakedness introduced by Nehring and Puppe (2007) is based on shortest paths: it requires that for every voter $i$ and for every alternative $x$, there is a shortest path between top $(i)$ and $x$ along which $i$ 's preferences are decreasing. (Without the word "shortest" this is equivalent to SPOC.) The impact of this alternative definition is that every voter's least-preferred alternative needs to be antipodal to the voter's peak; this is strictly more restrictive than SPOC. It would be interesting to see whether this smaller domain allows for a wider range of positive results than SPOC.

4. As PAV is based on dichotomous preferences, single-peakedness corresponds to the candidate interval restriction (Faliszewski et al., 2011; Elkind \& Lackner, 2015).

5. However, a recent study showed in numerical experiments that calculating OWA-based rules is computationally more expensive on random SPOC profiles than on random single-peaked profiles (Szufa, Faliszewski, Skowron, Slinko, \& Talmon, 2020). 
The SPOC domain is strictly larger the single-peaked domain. To quantify the actual gain in generality - or the increase in likelihood - it would be of interest to know the number of SPOC profiles for a given number of voters and candidates. This line of work has been pursued by Durand (2003), Lackner and Lackner (2017), and Chen and Finnendahl (2018) for single-peaked profiles and thus could be compared with results for SPOC. We note that there is a one-to-one correspondence between SPOC profiles with two voters and square permutations, ${ }^{6}$ which can be used to obtain enumerative results.

Another direction for future work would be to extend the SPOC concept to two (and more) dimensions - preferences single-peaked on a sphere - but this may be difficult since little is known even about extensions of single-peakedness on a line to two or more dimensions (see Sui, Francois-Nienaber, \& Boutilier, 2013). Further generalizations can be realised by considering almost SPOC profiles, mirroring the literature on almost single-peaked profiles (Elkind, Faliszewski, \& Slinko, 2012; Cornaz et al., 2012; Cornaz, Galand, \& Spanjaard, 2013; Faliszewski, Hemaspaandra, \& Hemaspaandra, 2014; Bredereck, Chen, \& Woeginger, 2016; Erdélyi, Lackner, \& Pfandler, 2017).

\section{Acknowledgements}

This paper is based on the two conference publications Preferences Single-Peaked on a Circle (Peters \& Lackner, 2017) and Single-Peakedness and Total Unimodularity: New Polynomial-Time Algorithms for Multi-Winner Elections (Peters, 2018).

We thank the anonymous reviewers of previous conference versions for useful suggestions and pointing out a mistake in one of our algorithms. We thank Edith Elkind for helpful discussions. Much of the work on this paper was performed while the authors were at the University of Oxford, supported by the European Research Council (ERC) under grant number 639945 (ACCORD). Martin Lackner was also supported by the Austrian Science Foundation FWF, grant P31890.

\section{Appendix A. Recognition Algorithm}

In this section, we describe the algorithm of Fitzsimmons and Lackner (2020) for deciding whether a profile consisting of weak orders, at least one of which is a linear order, is singlepeaked. This algorithm is the basis of Theorem 2 (showing that SPOC profiles of linear orders can be recognised in linear time) and is also the basis of the proof of Theorem 3 (characterisation by forbidden subprofiles), as we will see in Appendix B.

Algorithm 1 (also called the guided algorithm) takes as input a profile $P$ of weak orders, at least one of which (the guiding vote $v_{g}$ ) is a linear order. The algorithm then attempts to construct an axis on which $P$ is single-peaked. The axis is constructed from the outside in. The process is guided by $v_{g}$ : the algorithm scans the alternatives in the ranking $v_{g}$ from

6. This correspondence can be seen by using the connection between profiles with two voters and permutations (as described in Lackner \& Lackner, 2017). Condition 1 in Theorem 3 then corresponds to avoiding the set of permutations $\{14325,14352,15324,15342,24315,24351,25314,25341,41325,41352,42315$, 42351, 51324, 51342, 52314, 52341\}, which characterises square permutations (Waton, 2007; Duchi \& Poulalhon, 2008; Albert, Linton, Ruškuc, Vatter, \& Waton, 2011; Duchi, 2019). We thank Alexander Karpov for pointing out this connection. 
bottom to top, and decides at each step whether to place the alternative to the left or to the right.

Let us introduce some notation for understanding Algorithm 1. The algorithm labels alternatives so that $c_{m} \succ_{g} c_{m-1} \succ_{g} \cdots \succ_{g} c_{1}$. We write $C_{>_{i}}=\left\{c_{i+1}, \ldots, c_{m}\right\}$. The algorithm represents the axis under construction as a list of alternatives. For two disjoint lists $A_{L}, A_{R}$ of alternatives, $\left\langle A_{L}, A_{R}\right\rangle$ is the concatenation of the lists. Similarly, we write $\left\langle c_{i}, A_{R}\right\rangle$ and $\left\langle A_{L}, c_{i}\right\rangle$ for preprending and appending an alternative to a list. For a collection $A^{\prime}$ of alternatives, and a vote $v_{k}$, we write $\max _{k}\left(A^{\prime}\right)$ for an arbitrary alternative such that $\max _{k}\left(A^{\prime}\right) \succcurlyeq a$ for all $a \in A^{\prime}$, and we write $\min _{k}\left(A^{\prime}\right)$ for an arbitrary alternative such that $a \succcurlyeq \min _{k}\left(A^{\prime}\right)$ for all $a \in A^{\prime}$. When deciding how to place alternatives, the algorithm checks whether some of the conditions (R1), (R2), (L1), or (L2) apply to a specific voter $v_{k}$. These conditions are defined as follows:

$$
\begin{aligned}
& c_{i} \succ_{k} \min _{k}\left(C_{>i}\right) \quad \text { and } \max _{k}\left(A_{L}\right) \succ_{k} \min _{k}\left(C_{>i}\right) \\
& \max _{k}\left(C_{>i}\right) \succ_{k} c_{i} \text { and } \max _{k}\left(A_{R}\right) \succ_{k} c_{i} \\
& c_{i} \succ_{k} \min _{k}\left(C_{>i}\right) \text { and } \max _{k}\left(A_{R}\right) \succ_{k} \min _{k}\left(C_{>i}\right) \\
& \max _{k}\left(C_{>i}\right) \succ_{k} c_{i} \text { and } \max _{k}\left(A_{L}\right) \succ_{k} c_{i}
\end{aligned}
$$

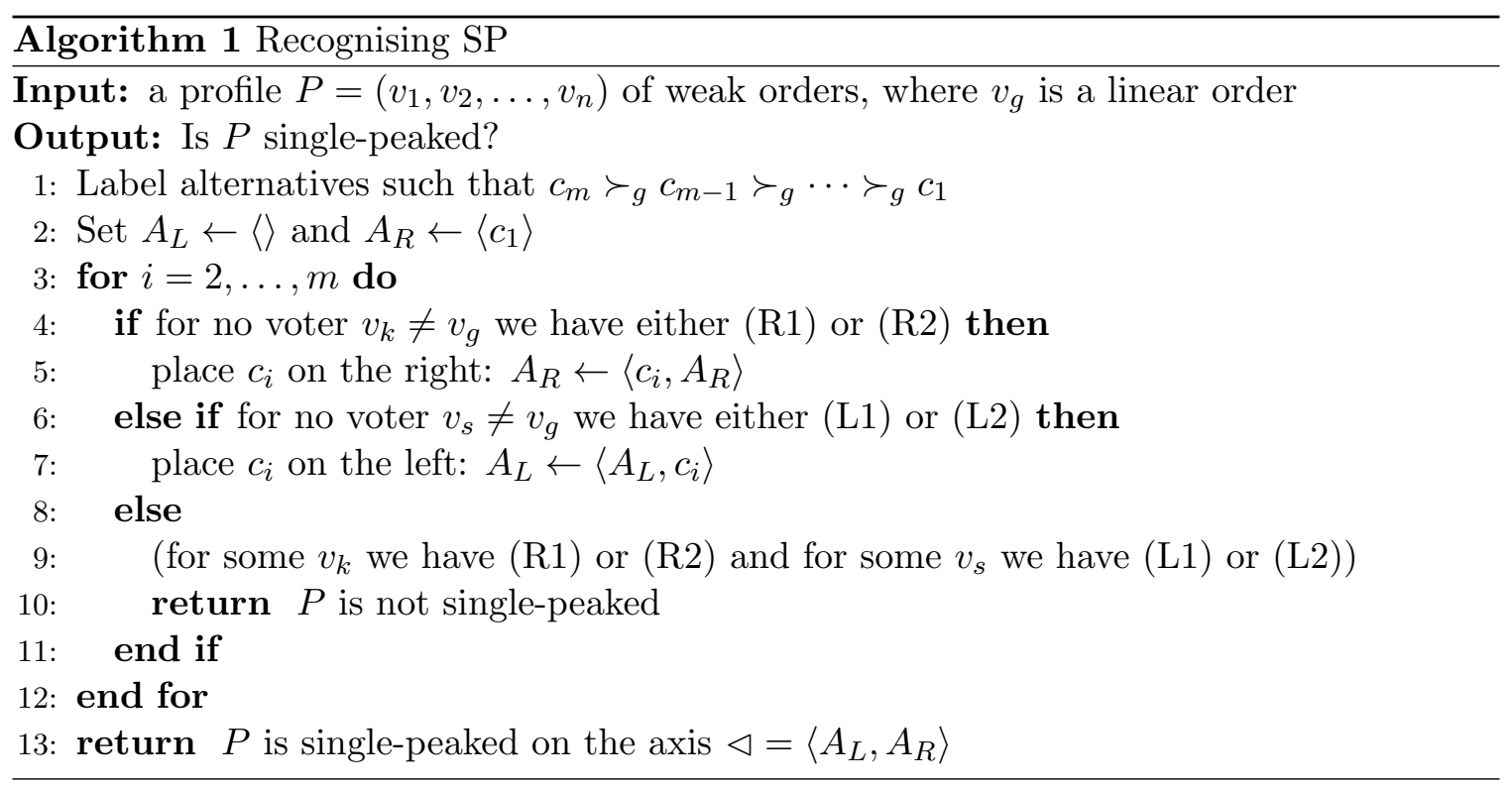

To prove that Algorithm 1 is correct, we need to prove that (i) when it returns an axis $\triangleleft$, then $P$ is single-peaked on $\triangleleft$, and that (ii) when it returns that $P$ is not singlepeaked, then $P$ is not single-peaked on any axis. This is shown by Fitzsimmons and Lackner (2020). Below, for completeness, we prove part (i). Part (ii) is not formally required for our purposes, since the correctness of the SPOC recognition algorithm only relies on (i).

Proposition 15 (Fitzsimmons \& Lackner, 2020). Let $P$ be a profile of weak orders, containing at least one linear order. If Algorithm 1 returns an axis $\triangleleft$, then $P$ is single-peaked on $\triangleleft$. 
Proof. It is easy to see that the guiding vote $v_{g}$ is single-peaked on $\triangleleft$, since any top-initial segment $\left\{c_{i}, c_{i+1}, \ldots, c_{m}\right\}$ of $v_{g}$ is an interval of $\triangleleft$ by construction. Suppose some other vote $v_{k} \neq v_{g}$ is not single-peaked on $\triangleleft$. Then there must be alternatives $a, b, c \in A$ such that $a \triangleleft b \triangleleft c$ and $a \succ_{k} b$ and $c \succ_{k} b$ (a valley).

We may assume without loss of generality that $c \succ_{g} a$ (otherwise we can rename alternatives so that the axis $\triangleleft$ is reversed). We perform a case analysis on the preferences of the guiding vote $v_{g}$, i.e., on the order in which Algorithm 1 has placed the alternatives:

- $a \prec_{g} b \prec_{g} c$ (alternatives are placed $a$ first then $b$ then $c$ ): Consider the iteration when $b$ was placed, i.e., when $c_{i}=b$. At this point, $a$ has already been placed, and since $a \triangleleft b \triangleleft c$, we have $a \in A_{L}$. Note that condition (L2) is now satisfied for $v_{k}$ since $a \succ_{k} b$ and $c \succ_{k} b$. Hence, $b$ must have been placed on the right. So the algorithm returns an axis with $a \triangleleft c \triangleleft b$, a contradiction.

- $a \prec_{g} c \prec_{g} b$ (alternatives are placed $a$ first then $c$ then $b$ ): Consider the iteration when $c$ was placed, i.e., when $c_{i}=c$. As before, we have $a \in A_{L}$. Note that (R1) is satisfied for $v_{k}$ and so $c$ is placed on the left. So the algorithm returns an axis with $a \triangleleft c \triangleleft b$, a contradiction.

- $b \prec_{g} a \prec_{g} c$ (alternatives are placed $b$ first then $a$ then $c$ ): If alternatives are placed in this order, the algorithm cannot obtain an axis with $a \triangleleft b \triangleleft c$, where $b$ is between $a$ and $c$, a contradiction.

Since each case leads to a contradiction, $v_{k}$ must in fact be single-peaked on $\triangleleft$.

\section{Appendix B. Characterisation by Forbidden Subprofiles}

Theorem 3. A profile $P$ of linear orders on $A$ is not SPOC if and only if one of the following three cases occurs.

1. There are distinct alternatives $a, b, c, d, e \in A$ and voters $v_{i}$ and $v_{j}$ in $P$ such that

$$
\begin{aligned}
& \{a, b\} \succ_{i}\{c\} \succ_{i}\{d, e\}, \\
& \{a, e\} \succ_{j}\{c\} \succ_{j}\{d, b\} .
\end{aligned}
$$

2. There are distinct alternatives $a, b, c, d \in A$ and voters $v_{i}, v_{j}$, and $v_{k}$ in $P$ such that

$$
\begin{aligned}
& \{a, b\} \succ_{i}\{c, d\}, \\
& \{a, c\} \succ_{j}\{b, d\}, \\
& \{a, d\} \succ_{k}\{b, c\} .
\end{aligned}
$$

3. There are distinct alternatives $a, b, c, d \in A$ and voters $v_{i}, v_{j}$, and $v_{k}$ in $P$ such that

$$
\begin{aligned}
& \{a, b\} \succ_{i}\{c, d\}, \\
& \{b, c\} \succ_{j}\{a, d\}, \\
& \{c, a\} \succ_{k}\{b, d\} .
\end{aligned}
$$


Proof. Sufficiency was proven in Section 4. Here, we prove necessity. Suppose that $P$ is a profile of linear orders which is not SPOC. Then the recognition algorithm of Theorem 2, run on input $P$, will return that $P$ is not SPOC (this follows from Propositions 1 and 15). We prove that if the algorithm gives a negative answer, then $P$ must contain one of the forbidden subprofiles.

The algorithm of Theorem 2 first constructs the profile $\bar{P}$ obtained from $P$ by slicing at some alternative $z$, where $z$ is an alternative which is ranked last by some voter (say $v_{1}$ ) in $P$. Then the upper part resulting from slicing $v_{1}$ is the same as $v_{1}$, and hence is a linear order. Call the voter in $\bar{P}$ corresponding to this upper part $g$. We label alternatives such that $c_{m} \succ_{g} c_{m-1} \succ_{g} \cdots \succ_{g} c_{2} \succ_{g} z$.

After constructing the sliced profile $\bar{P}$, the algorithm of Theorem 2 invokes Algorithm 1 from Appendix A and runs it on $\bar{P}$ to determine whether $\bar{P}$ is single-peaked. From Proposition 1, we know that $\bar{P}$ is single-peaked if and only if $P$ is SPOC. Since we assumed that $P$ is not SPOC, $\bar{P}$ is not single-peaked, and hence Algorithm 1 will return that $\bar{P}$ is not single-peaked (by Proposition 15). We will analyse all possible scenarios under which Algorithm 1 can give a negative answer, and show that in each case one of the three conditions of Theorem 3 applies. These conditions all refer to the profile $P$ rather than $\bar{P}$, and it will be convenient to transform them into statements about $\bar{P}$, which we do in two steps.

First, note that the conditions of Theorem 3 are closed under reversing some of the votes in the condition. We formalise this observation in Lemma 16 below. For a voter $v_{j}$, we write $x_{1} \leftrightarrow_{j} x_{2} \leftrightarrow_{j} \cdots \leftrightarrow_{j} x_{k}$ if either $x_{1} \succ_{j} x_{2} \succ_{j} \cdots \succ_{j} x_{k}$ or $x_{k} \succ_{j} \cdots \succ_{j} x_{2} \succ_{j} x_{1}$. Where we do not care about the relative order of some alternatives, we write sets as before: $A_{1} \leftrightarrow_{j} A_{2} \leftrightarrow_{j} A_{3}$ means that either $A_{1} \succ_{j} A_{2} \succ_{j} A_{3}$ or $A_{3} \succ_{j} A_{2} \succ_{j} A_{1}$.

Lemma 16. Condition 1 of Theorem 3 is satisfied if and only if there are distinct alternatives $a, b, c, d, e \in A$ and voters $v_{i}$ and $v_{j}$ in $P$ such that

$$
\begin{aligned}
& \{a, b\} \leftrightarrow_{i}\{c\} \leftrightarrow_{j}\{d, e\}, \\
& \{a, e\} \leftrightarrow_{j}\{c\} \leftrightarrow_{j}\{d, b\} .
\end{aligned}
$$

One of conditions 2 or 3 of Theorem 3 is satisfied if and only if there are distinct alternatives $a, b, c, d \in A$ and voters $v_{i}, v_{j}$, and $v_{k}$ in $P$ such that

$$
\begin{aligned}
& \{a, b\} \leftrightarrow_{i}\{c, d\}, \\
& \{a, c\} \leftrightarrow_{j}\{b, d\}, \\
& \{a, d\} \leftrightarrow_{k}\{b, c\} .
\end{aligned}
$$

Proof of Lemma 16. An easy case analysis.

Second, we give conditions on the sliced profile $\bar{P}$ whose occurrence implies that one of the conditions of Lemma 16 holds. From now on, we will label the voters as $\bar{P}=$ $\left(\bar{v}_{1}, \ldots, \bar{v}_{2 n}\right)$. The voter corresponding to the upper part of the guiding vote will also be called $g$. For a voter $\bar{v}_{i}$, we write $a \succ_{i} b$ if $a$ is ranked higher than $b$ in $\bar{v}_{i}$.

Lemma 17. One of the conditions of Theorem 3 (equivalently, of Lemma 16) is satisfied if one of the following sufficient conditions is satisfied: 
1. There are distinct alternatives $a, b, c, d \in A \backslash\{z\}$ and $e \in A$ and voters $\bar{v}_{i}$ and $\bar{v}_{j}$ in $\bar{P}$ such that

$$
\begin{aligned}
& \{a, b\} \succ_{i}\{c\} \succ_{i}\{e, d\} \\
& \{a, d\} \succ_{j}\{c\} \succ_{j}\{e, b\} .
\end{aligned}
$$

2. There are distinct alternatives $a, b, c, d \in A \backslash\{z\}$ and voters $\bar{v}_{i}, \bar{v}_{j}$, and $\bar{v}_{k}$ in $\bar{P}$ such that

$$
\begin{aligned}
& \{a, b\} \succ_{i}\{c, d\} \\
& \{a, c\} \succ_{j}\{b, d\} \\
& \{a, d\} \succ_{k}\{b, c\} .
\end{aligned}
$$

3. There are distinct alternatives $a, b, c \in A \backslash\{z\}$ and $d \in A$ and voters $\bar{v}_{i}, \bar{v}_{j}$, and $\bar{v}_{k}$ in $\bar{P}$ such that

$$
\begin{aligned}
& \{a, b\} \succ_{i}\{c, d\}, \\
& \{b, c\} \succ_{j}\{a, d\}, \\
& \{c, a\} \succ_{k}\{b, d\} .
\end{aligned}
$$

4. There are distinct alternatives $a, b, c, d \in A \backslash\{z\}$ and voters $\bar{v}_{i}$ and $\bar{v}_{j}$ in $\bar{P}$ such that

$$
\begin{aligned}
& \{a, b\} \succ_{i} c \sim_{i} d, \\
& \{a, c\} \succ_{j} b \sim_{i} d .
\end{aligned}
$$

5. There are distinct alternatives $a, b, c \in A \backslash\{z\}$ and voters $\bar{v}_{i}, \bar{v}_{j}$, and $\bar{v}_{k}$ in $\bar{P}$ such that

$$
\begin{gathered}
\{a, b\} \succ_{i}\{c, z\}, \\
\{a, c\} \succ_{j}\{b, z\}, \\
a \succ_{k} z \sim_{k} b \sim_{k} c .
\end{gathered}
$$

Proof of Lemma 17. We start with a useful observation: Suppose that $\bar{v}_{i}$ is a voter in $\bar{P}$ with $x_{1} \succ_{i} \cdots \succ_{i} x_{k}$ for some $x_{1}, \ldots, x_{k} \in A \backslash\{z\}$. Consider the voter $v_{i^{*}}$ in $P$ from which $\bar{v}_{i}$ was obtained. Then, for $v_{i^{*}}$, we have $x_{1} \leftrightarrow i_{i^{*}} \cdots \leftrightarrow_{i^{*}} x_{k} \leftrightarrow \leftrightarrow_{i^{*}} z$, by the definition of slicing.

Suppose conditions 1, 2, or 3 of this lemma hold. Then apply the above observation to each of the voters, obtaining one of the cases of Lemma 16.

Suppose condition 4 holds. Then consider the voters $v_{i^{*}}$ and $v_{j^{*}}$ in $P$ from which $\bar{v}_{i}$ and $\bar{v}_{j}$ were obtained. Because $\bar{v}_{i}$ is indifferent between $c$ and $d$, in the vote $v_{i^{*}}$, the alternatives $c$ and $d$ are separated from $a$ and $b$ by the alternative $z$ (by the definition of slicing). Thus, we have $\{a, b\} \leftrightarrow_{i^{*}} z \leftrightarrow_{i^{*}}\{c, d\}$. Similarly, we have $\{a, c\} \leftrightarrow_{j^{*}} z \leftrightarrow_{j^{*}}\{b, d\}$. Thus, by the first part of Lemma 16, we are done.

Suppose condition 5 hold. Consider the voters $v_{i^{*}}, v_{j^{*}}$, and $v_{k^{*}}$ in $P$ from which $\bar{v}_{i}, \bar{v}_{j}$, and $\bar{v}_{k}$ were obtained. Then we have $\{a, b\} \leftrightarrow_{i^{*}}\{c, z\}$ and $\{a, c\} \leftrightarrow_{j^{*}}\{b, z\}$. Further, in the vote $v_{k^{*}}$, the alternatives $b$ and $c$ are separated from $a$ by the alternative $z$, and thus $a \leftrightarrow k^{*} z \leftrightarrow_{k^{*}}\{b, z\}$. Thus, by the second part of Lemma 16, we are done. 
As we said above, because $P$ is not SPOC, $\bar{P}$ is not single-peaked, and hence Algorithm 1 returns a negative answer when run on $\bar{P}$. Thus, line 10 of Algorithm 1 is executed. Inspecting the if-clauses, this means that there is some iteration $i$ (in which we are placing alternative $c_{i}$ on the axis) when

for some $\bar{v}_{k}$ in $\bar{P}$ we have (R1) or (R2), and for some $\bar{v}_{s}$ in $\bar{P}$ we have (L1) or (L2).

By case analysis, we will prove that $(*)$ implies that one of the conditions of Lemma 17 holds. This suffices to prove the theorem. The cases we consider are:

A. condition R1 and L1 holding for the same voter $\left(\bar{v}_{k}=\bar{v}_{s}\right)$,

B. condition R2 and L2 holding for the same voter $\left(\bar{v}_{k}=\bar{v}_{s}\right)$,

C. condition R1 and L1 holding for different voters $\left(\bar{v}_{k} \neq \bar{v}_{s}\right)$,

D. condition R2 and L2 holding for different voters $\left(\bar{v}_{k} \neq \bar{v}_{s}\right)$,

E. condition R1 and L2 holding for the same voter $\left(\bar{v}_{k}=\bar{v}_{s}\right)$, and

F. condition R1 and L2 holding for different voters $\left(\bar{v}_{k} \neq \bar{v}_{s}\right)$.

Note that R1 and L1 are symmetric; the same holds for R2 and L2. Hence we can omit the analogues of cases $\mathrm{E}$ and $\mathrm{F}$ for condition R2 and L1.

For each of the cases $\mathrm{A}$ to $\mathrm{F}$, we need to identify voters and alternatives to which one of the conditions of Lemma 17 apply. To do so, we will repeatedly apply the following rules, which can be proven by inspecting Algorithm 1 . These rules are valid for every $\bar{v}_{i}$ in $\bar{P}$, and they hold at each iteration $i$ of Algorithm 1, with $A_{L}$ and $A_{R}$ as defined by the algorithm at the start of the iteration.

- (g) - We have $\left\{c_{i+1}, \ldots, c_{m}\right\} \succ_{g}\left\{c_{i}\right\} \succ_{g}\left(A_{L} \cup A_{R}\right) \backslash\{z\} \succ_{g}\{z\}$, by the choice of vote $g$, its role in the guiding algorithm, and our labelling of alternatives.

- (z) - Let $a, b \in A$. If $a \succ_{i} b$, then $a \succ_{i} z$. Furthermore, if $a \sim_{i} b$, then $a \sim_{i} z$. This is because $z$ is ranked last in all votes in $\bar{P}$ and $\bar{P}$ contains top orders.

- (top) - Let $a, b, c \in A$ with $a \neq b$. If $a \sim_{i} b$ and $c \succ_{i} a$, then $c \succ_{i} b$. This is because in all votes in $\bar{P}$, indifferences only occur among bottom-ranked alternatives (they are top orders).

- (ind) - Let $\ell \in A_{L}$ and $a \in\left\{c_{i}, \ldots, c_{m}\right\}$. If $\ell \succ_{i} a$, then $a \succcurlyeq_{i} r$ for all $r \in A_{R}$. Similarly if left and right are reversed. This follows from the inductive correctness proof of the guided algorithm; the algorithm would not have placed $\ell$ and $r$ in such a way that $\ell, a, r$ form a valley.

- (ind2) - Let $\ell_{1}, \ell_{2} \in A_{L}$ such that $\ell_{1}$ is placed left of $\ell_{2}$ in $A_{L}$, and let $a \in$ $\left\{c_{i}, \ldots, c_{m}\right\}$. Then $\ell_{2} \succcurlyeq_{i} \ell_{1}$ or $\ell_{2} \succcurlyeq_{i} a$. Similarly if left and right are reversed. This follows from the inductive correctness proof of the guided algorithm; otherwise the algorithm would not have placed $\ell_{2}$ on the left side as $\ell_{1}, \ell_{2}, a$ would form a valley. 
We will use pictures to display various relations between alternations. Arrows $a \rightarrow b$ signify that $a \succ b$; labels on arrows indicate the rule used to deduce this relation. Similarly, a dashed line $a---b$ indicates $a \sim b$.

We are now ready to go through our case analysis.

A. Condition R1 holds for voter $\bar{v}_{k}$, and condition L1 holds for voter $\bar{v}_{k}$.

This case is impossible due to (ind): we have both $\max _{k}\left(A_{L}\right) \succ_{k} \min _{k}\left(C_{>i}\right)$ as well as $\max _{k}\left(A_{R}\right) \succ_{k} \min _{k}\left(C_{>i}\right)$.

B. Condition R2 holds for voter $\bar{v}_{k}$, and condition L2 holds for voter $\bar{v}_{k}$.

This case is also impossible due to (ind): we have both $\max _{k}\left(A_{L}\right) \succ_{k} c_{i}$ and $\max _{k}\left(A_{R}\right) \succ_{k}$ $c_{i}$.

C. Condition R1 holds for voter $\bar{v}_{k}$, and condition L1 holds for voter $\bar{v}_{s}$, with $\bar{v}_{k} \neq \bar{v}_{s}$.

Let $w_{k}=\min _{k}\left(C_{>i}\right)$, let $w_{s}=\min _{s}\left(C_{>i}\right)$, let $\ell_{k}=\max _{k}\left(A_{L}\right)$, and let $r_{s}=\max _{s}\left(A_{R}\right)$.

By R1, $c_{i} \succ_{k} w_{k}$ and $\ell_{k} \succ_{k} w_{k}$. By L1, $c_{i} \succ_{s} w_{s}$ and $r_{s} \succ_{s} w_{s}$.

C.1. Suppose $w_{k}=w_{s}=: w$. From (ind), we have $w \succcurlyeq_{k} r_{s}$ and $w \succcurlyeq_{s} \ell_{k}$.

C.1.a. Suppose $w \succ_{k} r_{s}$ and $w \succ_{s} \ell_{k}$. Then the following relations can be deduced:

sliced vote $\bar{v}_{k}: \quad \quad$ sliced vote $\bar{v}_{s}$ :
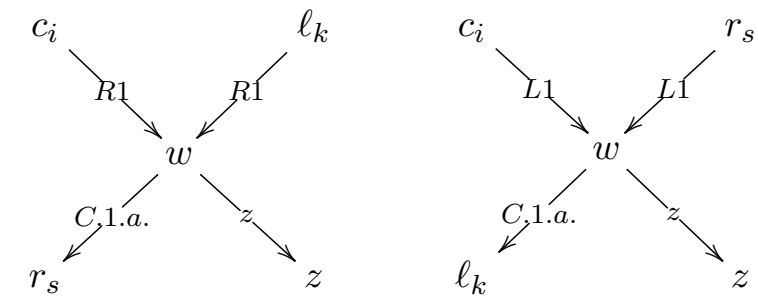

This corresponds to case 1 in Lemma 17 and thus we encounter case 1 in the theorem statement.

C.1.b. Suppose $w \sim_{k} r_{s}$ and $w \sim_{s} \ell_{k}$. Then

sliced vote $\bar{v}_{k}$ : $\quad$ sliced vote $\bar{v}_{s}$ :
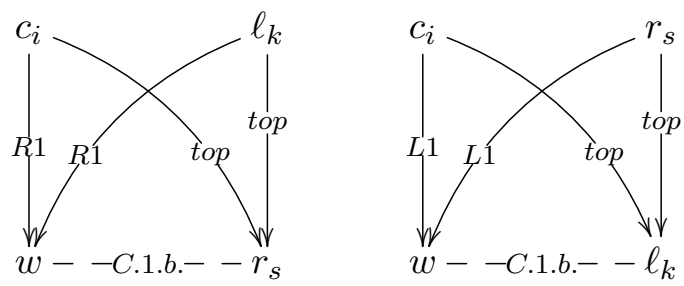

This corresponds to case 4 in Lemma 17.

C.1.c. Suppose $w \succ_{k} r_{s}$ and $w \sim_{s} \ell_{k}$. 
sliced vote $\bar{v}_{k}$ :

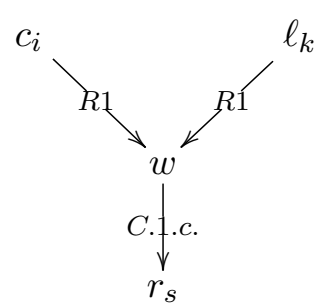

sliced vote $\bar{v}_{s}$ :

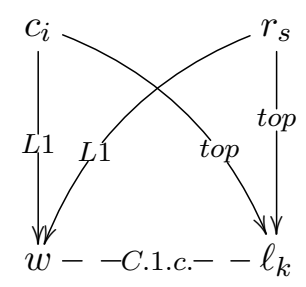

guiding vote $\bar{g}$ :

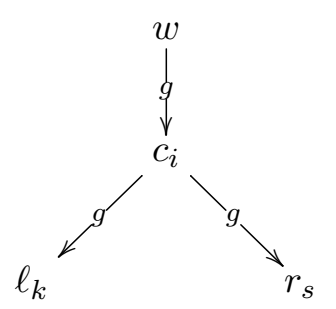

This corresponds to case 2 in Lemma 17.

C.1.d. Suppose $w \sim_{k} r_{s}$ and $w \succ_{s} \ell_{k}$. Then, symmetrically to C.1.c.,

sliced vote $\bar{v}_{k}$ :

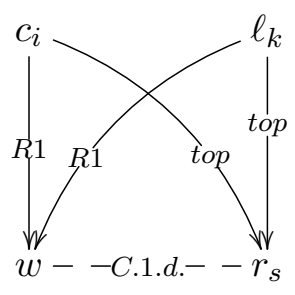

sliced vote $\bar{v}_{s}$ :

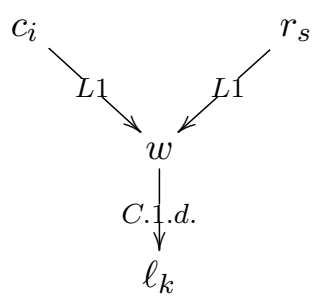

guiding vote $\bar{g}$ :

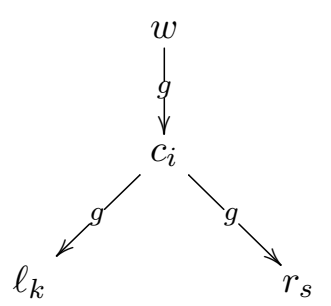

This corresponds to case 2 in Lemma 17.

C.2. Suppose $w_{k} \neq w_{s}$. By definition of $w_{s}$ and $w_{k}$, we have $w_{s} \succcurlyeq_{k} w_{k}$ and $w_{k} \succcurlyeq_{s} w_{s}$.

C.2.a. Suppose $w_{s} \succ_{k} w_{k}$ and $w_{k} \succ_{s} w_{s}$. Then we have

sliced vote $\bar{v}_{k}$ :

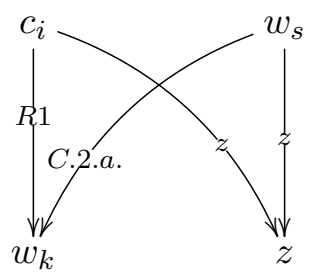

sliced vote $\bar{v}_{s}$ :

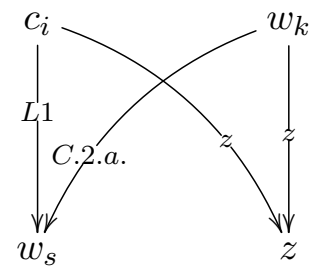

guiding vote $\bar{g}$ :

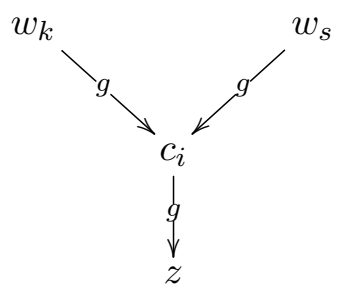

This corresponds to case 3 in Lemma 17.

C.2.b. Suppose $w_{s} \sim_{k} w_{k}$ and $w_{k} \sim_{s} w_{s}$. Then we have

sliced vote $\bar{v}_{k}$ :

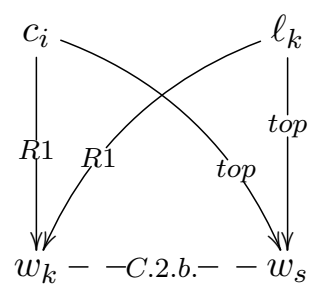

sliced vote $\bar{v}_{s}$ :

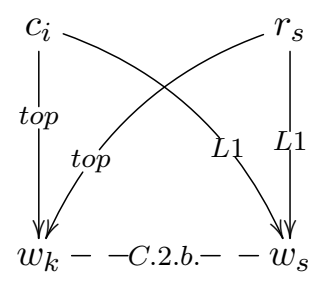

This corresponds to case 4 in Lemma 17. 
C.2.c. Suppose $w_{s} \succ_{k} w_{k}$ and $w_{k} \sim_{s} w_{s}$. Since $\ell_{k} \succ_{k} w_{k}$ by R1, we have $w_{k} \succcurlyeq_{k} r_{s}$ by (ind), and thus $\ell_{k} \succ_{k} r_{s}$ by transitivity. Similarly we obtain the other arrows labelled (ind) below, each involving a use of transitivity.

sliced vote $\bar{v}_{k}$ :

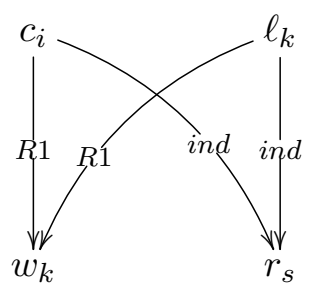

sliced vote $\bar{v}_{s}$ :

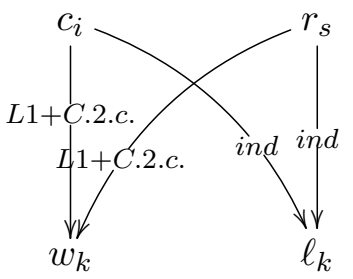

guiding vote $\bar{g}$ :

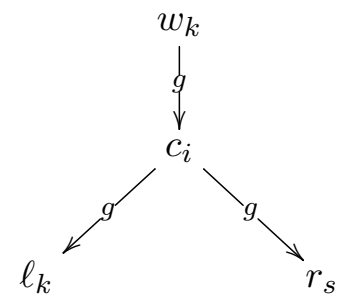

This corresponds to case 2 in Lemma 17. The arrows labeled with "L1+C.2.c." are obtained by using L1 for $c_{i} \succ_{s} w_{s}$ and $r_{s} \succ_{s} w_{s}$ and the assumption of case C.2.c., $w_{k} \sim_{s} w_{s}$.

C.2.d. Suppose $w_{s} \sim_{k} w_{k}$ and $w_{k} \succ_{s} w_{s}$. Then, symmetric to 2.c.,

sliced vote $\bar{v}_{k}$ :

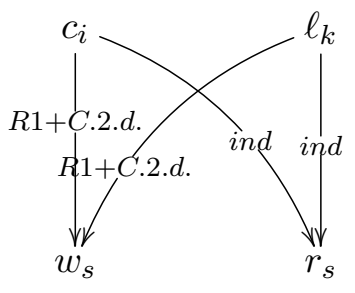

sliced vote $\bar{v}_{s}$ :

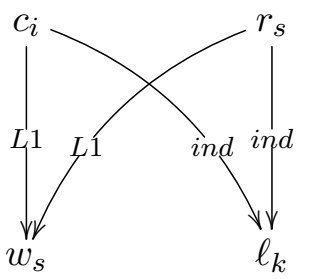

guiding vote $\bar{g}$ :

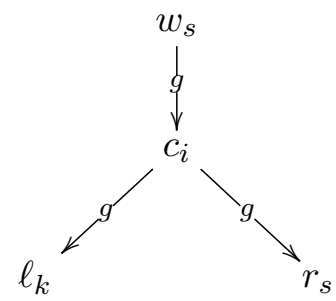

This corresponds to case 2 in Lemma 17.

D. Condition R2 holds for voter $\bar{v}_{k}$, and condition L2 holds for voter $\bar{v}_{s}$, with $\bar{v}_{k} \neq \bar{v}_{s}$.

Let $b_{k}=\max _{k}\left(C_{>i}\right)$, let $b_{s}=\max _{s}\left(C_{>i}\right)$, let $\ell_{k}=\max _{k}\left(A_{L}\right)$, and let $r_{s}=\max _{s}\left(A_{R}\right)$.

By R2, $b_{k} \succ_{k} c_{i}$ and $\ell_{k} \succ_{k} c_{i}$. By L2, $b_{s} \succ_{s} c_{i}$ and $r_{s} \succ_{s} c_{i}$.

D.1. Suppose $b_{k}=b_{s}=: b$. The case D.1. is similar to case C.1.; we present it for completeness.

D.1.a. Suppose $c_{i} \succ_{k} r_{s}$ and $c_{i} \succ_{s} \ell_{k}$. Then:

$$
\text { sliced vote } \bar{v}_{k} \text { : } \quad \text { sliced vote } \bar{v}_{s} \text { : }
$$
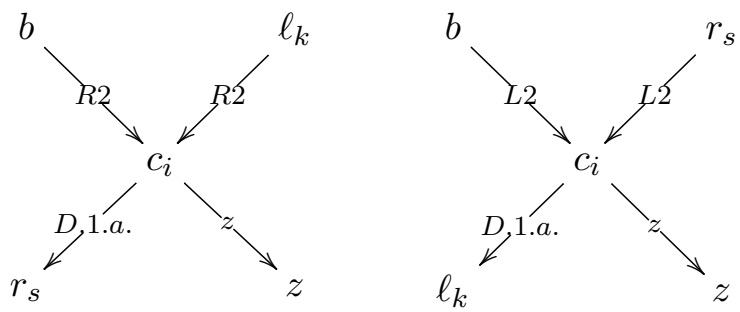

This corresponds to case 1 in Lemma 17. 
D.1.b. Suppose $c_{i} \sim_{k} r_{s}$ and $c_{i} \sim_{s} \ell_{k}$. Then

sliced vote $\bar{v}_{k}: \quad \quad$ sliced vote $\bar{v}_{s}$ :
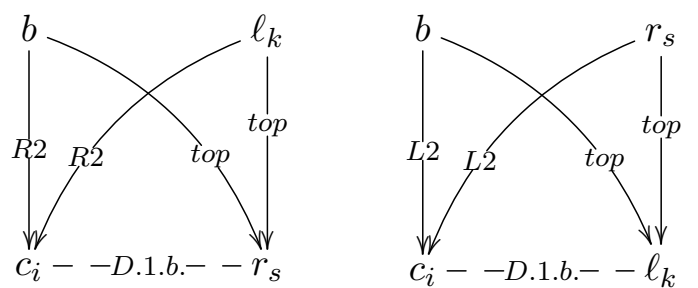

This corresponds to case 4 in Lemma 17.

D.1.c. Suppose $c_{i} \succ_{k} r_{s}$ and $c_{i} \sim_{s} \ell_{k}$.

sliced vote $\bar{v}_{k}$ :

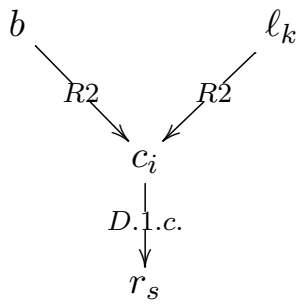

sliced vote $\bar{v}_{s}$ :

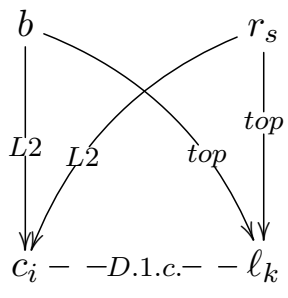

guiding vote $\bar{g}$ :

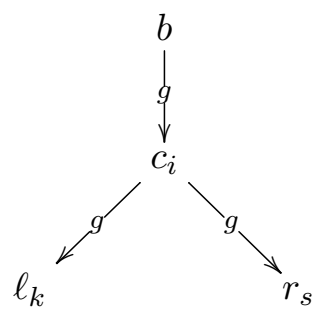

This corresponds to case 2 in Lemma 17.

D.1.d. Suppose $c_{i} \sim_{k} r_{s}$ and $c_{i} \succ_{s} \ell_{k}$. This case is symmetric to D.1.c.

D.2. Suppose $b_{k} \neq b_{s}$.

D.2.a. Suppose that either (i) $c_{i} \succ_{k} b_{s}$ or (ii) $c_{i} \succ_{s} b_{k}$. Then we can deduce one of the first two depicted relations $\left(\bar{v}_{k}\right.$ or $\left.\bar{v}_{s}\right)$ as well as the guiding vote $\bar{g}$ :

sliced vote $\bar{v}_{k}: \quad$ sliced vote $\bar{v}_{s}: \quad$ guiding vote $\bar{g}$ :
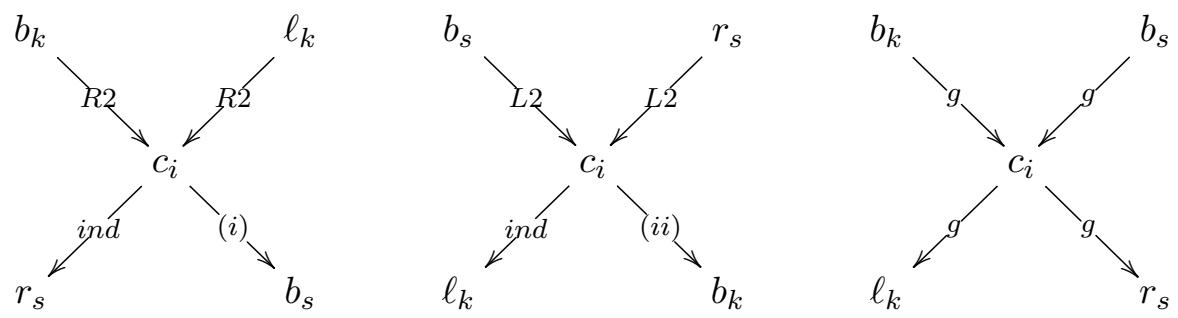

If $c_{i} \succ_{k} b_{s}$, then $k$ and $g$ correspond to case 1 in Lemma 17; if $c_{i} \succ_{s} b_{k}$, then $s$ and $g$ correspond to case 1 in Lemma 17.

D.2.b. Now suppose that (i) $c_{i} \prec_{k} b_{s}$ or (ii) $c_{i} \prec_{s} b_{k}$. As $\ell_{k} \succ_{k} c_{i}$ (from R2), by (ind) we have $c_{i} \succeq_{k} r_{s}$. Analogously, since $r_{s} \succ_{s} c_{i}$ (from L2), by (ind) we have $c_{i} \succeq_{s} \ell_{k}$. Thus: 
sliced vote $\bar{v}_{k}$ :

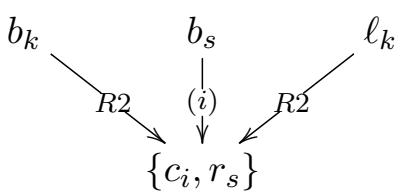

sliced vote $\bar{v}_{s}$ :

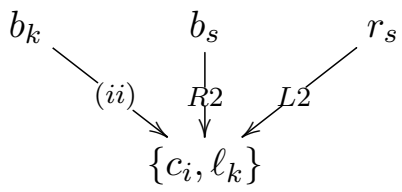

guiding vote $\bar{g}$ :

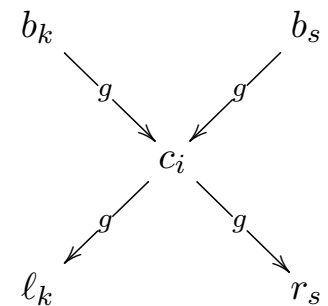

In case (i), this corresponds to case 2 in Lemma 17 when considering $\left\{b_{s}, c_{i}, \ell_{k}, r_{s}\right\}$. In case (ii), this corresponds to case 2 in Lemma 17 when considering $\left\{b_{k}, c_{i}, \ell_{k}, r_{s}\right\}$.

D.2.c. Otherwise, we have $c_{i} \sim_{k} b_{s}$ and $c_{i} \sim_{s} b_{k}$. By (ind), from $\ell_{k} \succ_{k} c_{i}$ (R2), and the fact that $\bar{v}_{k}$ is a top order, we have $c_{i} \sim_{k} b_{s} \sim_{k} r_{s}$. Analogously, by (ind), from $r_{s} \succ_{s} c_{i}(\mathrm{~L} 2)$, and the fact that $\bar{v}_{s}$ is a top order, we have $c_{i} \sim_{s} b_{k} \sim_{s} \ell_{k}$.

sliced vote $\bar{v}_{k}: \quad \quad$ sliced vote $\bar{v}_{s}$ :
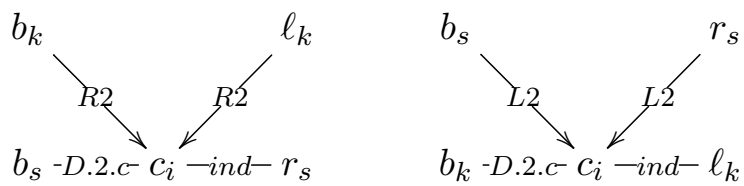

We see that $\bar{v}_{k}$ and $\bar{v}_{s}$ hold little information. Thus, we consider their opposite sliced part, i.e., if $\bar{v}_{k}$ is a sliced upper part, then we consider the lower part, or vice versa. Let $\bar{v}_{k}^{\prime}$ and $\bar{v}_{s}^{\prime}$ be the corresponding opposite parts.

Now suppose that a candidate $y \neq z$ is in the lowest level of $\bar{v}_{k}$. Then $y$ is not in the lowest level of $\bar{v}_{k}^{\prime}$. Also, if $x \succ_{k} y$, then $x$ is in the lowest level of $\bar{v}_{k}^{\prime}$, and hence $y \succ_{k^{\prime}} x$. Call this observation (opp). From this we get:

opposite sliced vote $\bar{v}_{k}^{\prime}: \quad$ opposite sliced vote $\bar{v}_{s}^{\prime}: \quad$ guiding vote $\bar{g}$ :
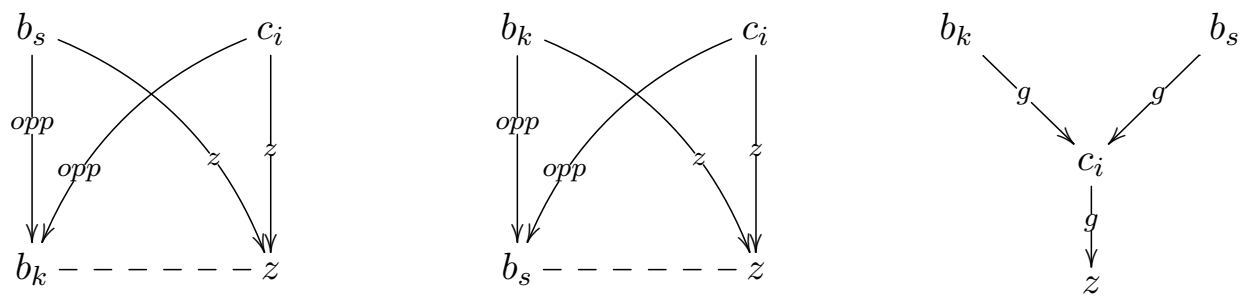

This corresponds to case 3 in Lemma 17.

E. Condition R1 holds for voter $\bar{v}_{k}$, and condition L2 holds for voter $\bar{v}_{k}$.

Let $w=\min _{k}\left(C_{>i}\right)$, let $b=\max _{k}\left(C_{>i}\right)$, let $\ell=\max _{k}\left(A_{L}\right)$.

By R1, $c_{i} \succ_{k} w$ and $\ell \succ_{k} w$. By L2, $b \succ_{k} c_{i}$ and $\ell \succ_{k} c_{i}$. Note that $b \succ_{k} c_{i} \succ_{k} w$ and so $b \neq w$. We have 
sliced vote $\bar{v}_{k}: \quad$ guiding vote $\bar{g}$ :
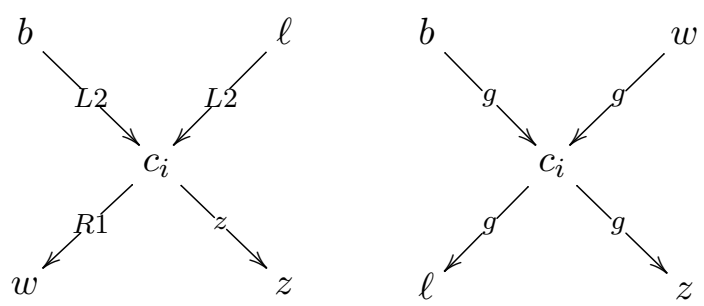

This corresponds to case 1 in Lemma 17.

F. Condition R1 holds for voter $\bar{v}_{k}$, and condition L2 holds for voter $\bar{v}_{s}$, with $\bar{v}_{k} \neq \bar{v}_{s}$.

Let $w_{k}=\min _{k}\left(C_{>i}\right)$, let $b_{s}=\max _{s}\left(C_{>i}\right)$, let $\ell_{k}=\max _{k}\left(A_{L}\right)$, and let $\ell_{s}=\max _{s}\left(A_{L}\right)$.

By R1, $c_{i} \succ_{k} w_{k}$ and $\ell_{k} \succ_{k} w_{k}$. By L2, $b_{s} \succ_{s} c_{i}$ and $\ell_{s} \succ_{s} c_{i}$.

F.1. Suppose $c_{i} \succ_{s} w_{k}$. Since then $b_{s} \succ_{s} c_{i} \succ_{s} w_{k}$, we have $b_{s} \neq w_{k}$. Then we have sliced vote $\bar{v}_{s}$ : guiding vote $\bar{g}$ :
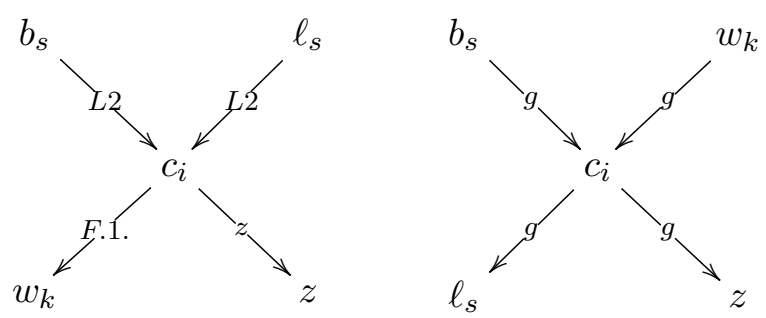

This corresponds to case 1 in Lemma 17.

F.2. Suppose $w_{k} \sim_{s} c_{i}$. Since $\bar{v}_{s}$ is a top order, and $b_{s} \succ_{s} c_{i} \sim_{s} w_{k}$, we must have $b_{s} \neq w_{k}$.

F.2.a. Suppose $b_{s} \succ_{k} w_{k}$. Then we have

sliced vote $\bar{v}_{k}$ :

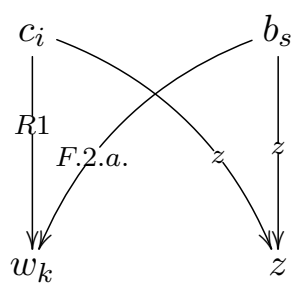

sliced vote $\bar{v}_{s}$ :

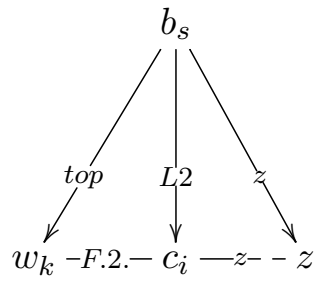

guiding vote $\bar{g}$ :

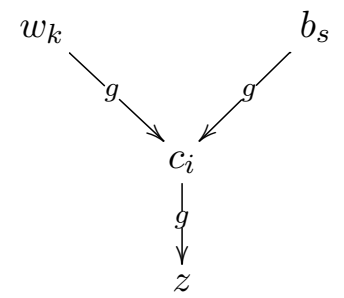

This corresponds to case 5 in Lemma 17.

F.2.b. Suppose $w_{k} \sim_{k} b_{s}$.

Claim: There is $\ell \in\left\{\ell_{s}, \ell_{k}\right\}$ for which both $\bar{v}_{s}$ and $\bar{v}_{k}$ agree that $\ell$ is not in the bottom layer

If $\ell_{s}=\ell_{k}$, we are done by R1 and L2. So suppose $\ell_{s} \neq \ell_{k}$. Now, by choice of $\ell_{s}$ and $\ell_{k}$, we have $\ell_{s} \succcurlyeq_{s} \ell_{k}$ and $\ell_{k} \succcurlyeq_{k} \ell_{s}$. But $\bar{v}_{s}$ and $\bar{v}_{k}$ are top orders, and $\ell_{s} \succ_{s} c_{i}$ and $\ell_{k} \succ_{k} w_{k}$, so actually $\ell_{s} \succ_{s} \ell_{k}$ and $\ell_{k} \succ_{k} \ell_{s}$. 
Next we show that it is not possible that both $\ell_{k}$ is in $s$ 's bottom layer and $\ell_{s}$ is in $k$ 's bottom layer. Suppose this was so. Suppose $\ell_{s}$ is placed by the algorithm to the left of $\ell_{k}$. Then $\bar{v}_{s}$ has a valley: $\ell_{s} \succ_{s} \ell_{k} \prec_{s} b_{s}$. Otherwise, $\ell_{k}$ is placed by the algorithm to the left of $\ell_{s}$. Then $\bar{v}_{k}$ has a valley: $\ell_{k} \succ_{k} \ell_{s} \prec_{k} c_{i}$. Both possibilities contradict (ind2).

With this choice of $\ell$, we have

sliced vote $\bar{v}_{k}: \quad \quad$ sliced vote $\bar{v}_{s}:$

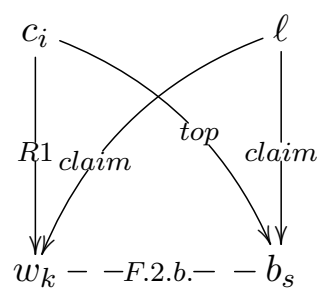

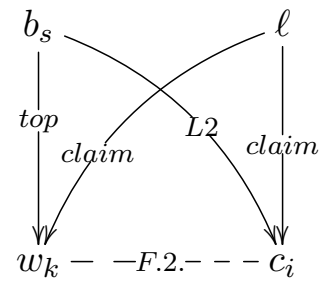

This corresponds to case 4 in Lemma 17.

F.2.c. Suppose $w_{k} \succ_{k} b_{s}$. This is impossible by choice of $w_{k}=\min _{k}\left(C_{>i}\right)$.

F.3. Suppose $w_{k} \succ_{s} c_{i}$. (Note we do not consider $b_{s}$ in the following subcases, so it does not matter if $b_{s}=w_{k}$ or not.)

F.3.a. Suppose $\ell_{s}=\ell_{k}=: \ell$. Then we have

sliced vote $\bar{v}_{k}: \quad \quad$ sliced vote $\bar{v}_{s}: \quad$ guiding vote $\bar{g}$ :
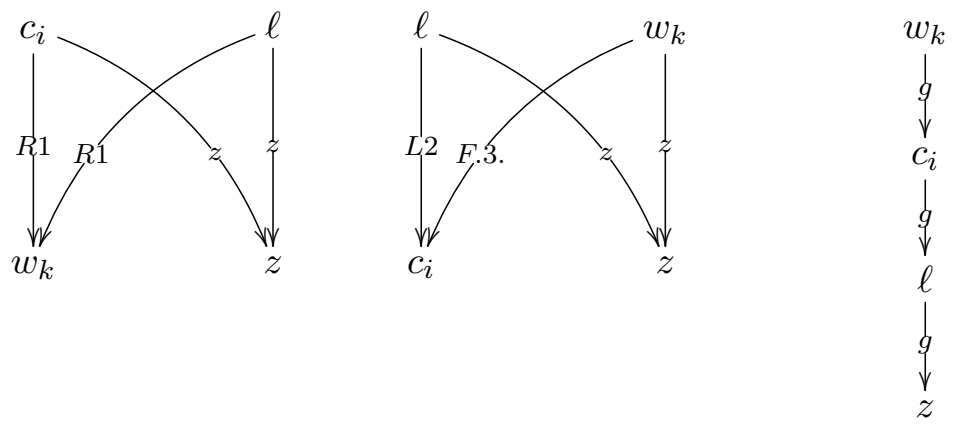

This corresponds to case 3 in Lemma 17.

F.3.b. Suppose $\ell_{k}$ lies to the left of $\ell_{s}$ (within $A_{L}$ ). By (ind2) it holds that $\ell_{s} \succcurlyeq_{k} \ell_{k}$ or $\ell_{s} \succcurlyeq_{k} c_{i}$. As $\ell_{k} \succ_{k} w_{k}$ and $c_{i} \succ_{k} w_{k}$ (both by R1), we can conclude that $\ell_{s} \succ_{k} w_{k}$. So we have 


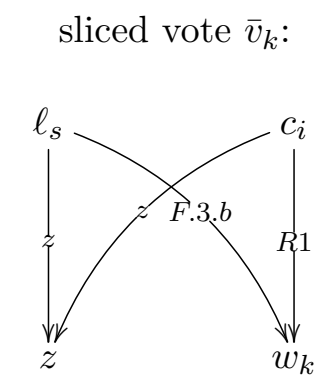

$$
\text { sliced vote } \bar{v}_{s} \text { : }
$$

guiding vote $\bar{g}$ :
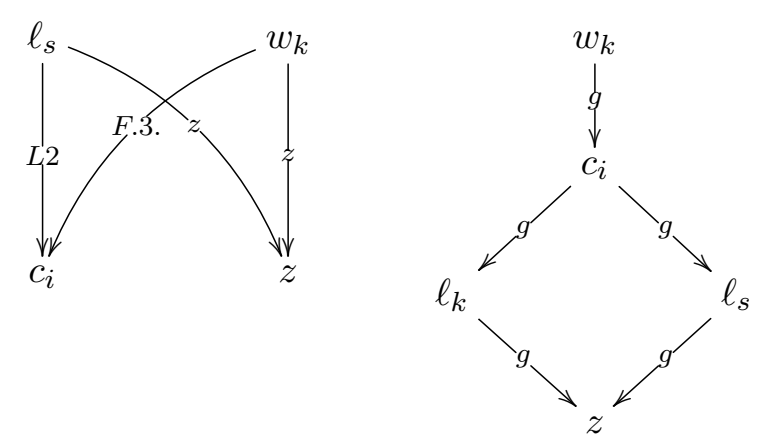

This corresponds to case 3 in Lemma 17.

F.3.c. Suppose $\ell_{s}$ lies to the left of $\ell_{k}$ (within $A_{L}$ ). By (ind2) it holds that $\ell_{k} \succeq_{s} w_{k}$ or $\ell_{k} \succeq_{s} \ell_{s}$. As $w_{k} \succ_{s} c_{i}$ (by F.3.) and $\ell_{s} \succ_{s} c_{i}$ (by L2), we can conclude that $\ell_{k} \succ_{s} c_{i}$. So we have

sliced vote $\bar{v}_{k}$ :

$$
\text { sliced vote } \bar{v}_{s} \text { : }
$$

guiding vote $\bar{g}$ :
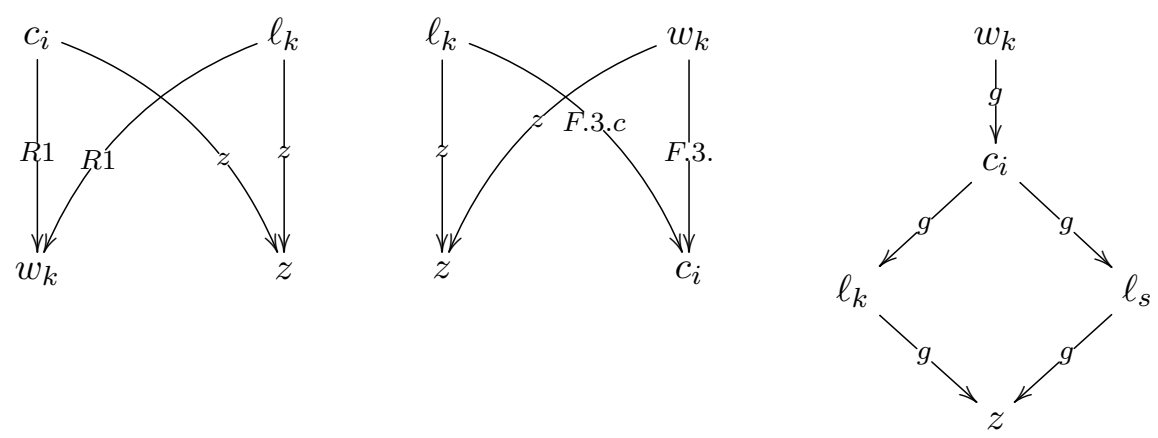

This corresponds to case 3 in Lemma 17.

We have shown that in all possible situations where the guided algorithm may return no, Lemma 17 is applicable and thus the theorem holds.

\section{References}

Achuthankutty, G., \& Roy, S. (2018). Dictatorship on top-circular domains. Theory and Decision, 85(3-4), 479-493.

Albert, M. H., Linton, S., Ruškuc, N., Vatter, V., \& Waton, S. (2011). On convex permutations. Discrete Mathematics, 311(8-9), 715-722.

Alcalde-Unzu, J., \& Vorsatz, M. (2018). Strategy-proof location of public facilities. Games and Economic Behavior, 112, 21-48.

Alon, N., Feldman, M., Procaccia, A. D., \& Tennenholtz, M. (2010a). Strategyproof approximation of the minimax on networks. Mathematics of Operations Research, 35(3), $513-526$.

Alon, N., Feldman, M., Procaccia, A. D., \& Tennenholtz, M. (2010b). Walking in circles. Discrete Mathematics, 310(23), 3432-3435. 
Aziz, H., Brill, M., Conitzer, V., Elkind, E., Freeman, R., \& Walsh, T. (2017). Justified representation in approval-based committee voting. Social Choice and Welfare, 48(2), $461-485$.

Ballester, M. A., \& Haeringer, G. (2011). A characterization of the single-peaked domain. Social Choice and Welfare, 36(2), 305-322.

Bartholdi III, J., Tovey, C. A., \& Trick, M. A. (1989). Voting schemes for which it can be difficult to tell who won the election. Social Choice and Welfare, 6(2), 157-165.

Bartholdi III, J., \& Trick, M. A. (1986). Stable matching with preferences derived from a psychological model. Operation Research Letters, 5(4), 165-169.

Betzler, N., Slinko, A., \& Uhlmann, J. (2013). On the computation of fully proportional representation. Journal of Artificial Intelligence Research, 47, 475-519.

Black, D. (1948). On the rationale of group decision-making. Journal of Political Economy, $56(1), 23-34$.

Booth, K. S., \& Lueker, G. S. (1976). Testing for the consecutive ones property, interval graphs, and graph planarity using PQ-tree algorithms. Journal of Computer and System Sciences, 13(3), 335-379.

Brandt, F., Geist, C., \& Peters, D. (2017). Optimal bounds for the no-show paradox via SAT solving. Mathematical Social Sciences, 90, 18-27.

Brandt, F., Brill, M., \& Harrenstein, P. (2016). Tournament solutions. In Brandt, F., Conitzer, V., Endriss, U., Lang, J., \& Procaccia, A. (Eds.), Handbook of Computational Social Choice. Cambridge University Press.

Brandt, F., Brill, M., Hemaspaandra, E., \& Hemaspaandra, L. A. (2015). Bypassing combinatorial protections: Polynomial-time algorithms for single-peaked electorates. Journal of Artificial Intelligence Research, 53, 439-496.

Bredereck, R., Chen, J., \& Woeginger, G. J. (2013). A characterization of the single-crossing domain. Social Choice and Welfare, 41(4), 989-998.

Bredereck, R., Chen, J., \& Woeginger, G. J. (2016). Are there any nicely structured preference profiles nearby?. Mathematical Social Sciences, 79, 61-73.

Chamberlin, B., \& Courant, P. (1983). Representative deliberations and representative decisions: Proportional representation and the Borda rule. American Political Science Review, 77 (3), 718-733.

Chebotarev, P. Y., \& Shamis, E. (1998). Characterizations of scoring methods for preference aggregation. Annals of Operations Research, 80, 299-332.

Chen, J., \& Finnendahl, U. P. (2018). On the number of single-peaked narcissistic or singlecrossing narcissistic preference profiles. Discrete Mathematics, 341(5), 1225-1236.

Chen, J., Pruhs, K. R., \& Woeginger, G. J. (2017). The one-dimensional Euclidean domain: finitely many obstructions are not enough. Social Choice and Welfare, 48(2), 409-432.

Cornaz, D., Galand, L., \& Spanjaard, O. (2012). Bounded single-peaked width and proportional representation. In Proceedings of the 20th European Conference on Artificial Intelligence (ECAI), pp. 270-275. 
Cornaz, D., Galand, L., \& Spanjaard, O. (2013). Kemeny elections with bounded singlepeaked or single-crossing width. In Proceedings of the 23rd International Joint Conference on Artificial Intelligence (IJCAI), pp. 76-82.

Debord, B. (1987). Caractérisation des matrices des préférences nettes et méthodes d'agrégation associées. Mathématiques et sciences humaines, 97, 5-17.

Demange, G. (1982). Single-peaked orders on a tree. Mathematical Social Sciences, 3(4), 389-396.

Dom, M. (2009). Algorithmic aspects of the consecutive-ones property. Bulletin of the European Association for Theoretical Computer Science, 98, 27-59.

Duchi, E. (2019). A code for square permutations and convex permutominoes. Discrete Mathematics \& Theoretical Computer Science, 21(2). Permutation Patterns 2018, arXiv:1904.02691.

Duchi, E., \& Poulalhon, D. (2008). On square permutations. In DMTCS Proceedings of the 5th Colloquium on Mathematics and Computer Science, pp. 207-222.

Durand, S. (2003). Finding sharper distinctions for conditions of transitivity of the majority method. Discrete Applied Mathematics, $131(3)$, 577-595.

Elkind, E., Faliszewski, P., \& Slinko, A. (2012). Clone structures in voters' preferences. In Proceedings of the 13th ACM Conference on Electronic Commerce (ACM-EC), pp. 496-513.

Elkind, E., \& Lackner, M. (2015). Structure in dichotomous preferences. In Proceedings of the 24th International Joint Conference on Artificial Intelligence (IJCAI), pp. 20192025.

Elkind, E., Lackner, M., \& Peters, D. (2016). Preference restrictions in computational social choice: Recent progress. In Proceedings of the 25th International Joint Conference on Artificial Intelligence (IJCAI), pp. 4062-4065.

Elkind, E., Lackner, M., \& Peters, D. (2017). Structured preferences. In Endriss, U. (Ed.), Trends in Computational Social Choice, chap. 10, pp. 187-207. AI Access.

Erdélyi, G., Lackner, M., \& Pfandler, A. (2017). Computational aspects of nearly singlepeaked electorates. Journal of Artificial Intelligence Research, 58, 297-337.

Faliszewski, P., Hemaspaandra, E., \& Hemaspaandra, L. (2014). The complexity of manipulative attacks in nearly single-peaked electorates. Artificial Intelligence, 207, 69-99.

Faliszewski, P., Skowron, P., Slinko, A., \& Talmon, N. (2017). Multiwinner rules on paths from $k$-Borda to Chamberlin-Courant. In Proceedings of the 25th International Joint Conference on Artificial Intelligence (IJCAI), pp. 192-198.

Faliszewski, P., Hemaspaandra, E., Hemaspaandra, L. A., \& Rothe, J. (2011). The shield that never was: Societies with single-peaked preferences are more open to manipulation and control. Information and Computation, 209(2), 89-107.

Faliszewski, P., Skowron, P., Slinko, A., \& Talmon, N. (2017). Multiwinner voting: A new challenge for social choice theory. In Endriss, U. (Ed.), Trends in Computational Social Choice, chap. 2, pp. 27-47. AI Access. 
Faliszewski, P., Szufa, S., \& Talmon, N. (2018). Optimization-based voting rule design: The closer to utopia the better. In Proceedings of the 17th International Conference on Autonomous Agents and Multiagent Systems (AAMAS), pp. 32-40.

Feigenbaum, I., \& Sethuraman, J. (2015). Strategyproof mechanisms for one-dimensional hybrid and obnoxious facility location models. In Incentive and Trust in E-Communities: Papers from the 2015 AAAI Workshop. arXiv:1412.3414.

Fischer, F., Hudry, O., \& Niedermeier, R. (2016). Weighted tournament solutions. In Brandt, F., Conitzer, V., Endriss, U., Lang, J., \& Procaccia, A. (Eds.), Handbook of Computational Social Choice. Cambridge University Press.

Fitzsimmons, Z., \& Hemaspaandra, E. (2020). Election score can be harder than winner. In Proceedings of the 24th European Conference on Artificial Intelligence (ECAI). To appear.

Fitzsimmons, Z., \& Lackner, M. (2020). Incomplete preferences in single-peaked electorates. Journal of Artificial Intelligence Research (JAIR), 67, 797-833.

Fluschnik, T., Skowron, P., Triphaus, M., \& Wilker, K. (2019). Fair knapsack. In Proceedings of the 33rd AAAI Conference on Artificial Intelligence (AAAI), pp. 1941-1948.

Hemaspaandra, E., Spakowski, H., \& Vogel, J. (2005). The complexity of Kemeny elections. Theoretical Computer Science, 349(3), 382-391.

Hoffman, A. J., \& Kruskal, J. B. (1956). Integral boundary points of convex polyhedra. In Kuhn, H. W., \& Tucker, A. W. (Eds.), Linear Inequalities and Related Systems, pp. 223-246. Princeton University Press.

Janson, S. (2016). Phragmén's and Thiele's election methods. Tech. rep. arXiv:1611.08826 [math.HO], arXiv.org.

Kim, K. H., \& Roush, F. W. (1980). Special domains and nonmanipulability. Mathematical Social Sciences, 1(1), 85-92.

Lackner, M., \& Skowron, P. (2018). Consistent approval-based multi-winner rules. In Proceedings of the 19th ACM Conference on Economics and Computation (ACM-EC), pp. 47-48. arXiv:1704.02453.

Lackner, M.-L., \& Lackner, M. (2017). On the likelihood of single-peaked preferences. Social Choice and Welfare, 48(4), 717-745.

Lackner, M. (2014). Incomplete preferences in single-peaked electorates. In Proceedings of the 28th AAAI Conference on Artificial Intelligence (AAAI), pp. 742-748.

Lu, T., \& Boutilier, C. (2011). Budgeted social choice: From consensus to personalized decision making. In Proceedings of the 22nd International Joint Conference on Artificial Intelligence (IJCAI), pp. 280-286.

Maurras, J. F., Truemper, K., \& Akgül, M. (1981). Polynomial algorithms for a class of linear programs. Mathematical Programming, 21(1), 121-136.

McGarvey, D. C. (1953). A theorem on the construction of voting paradoxes. Econometrica, $21(4), 608-610$.

Moulin, H. (1991). Axioms of Cooperative Decision Making. Cambridge University Press. 
Moulin, H. (1988). Condorcet's principle implies the no show paradox. Journal of Economic Theory, 45(1), 53-64.

Nehring, K., \& Puppe, C. (2007). The structure of strategy-proof social choice, Part I: General characterization and possibility results on median spaces. Journal of Economic Theory, 135(1), 269-305.

Peters, D. (2017). Recognising multidimensional Euclidean preferences. In Proceedings of the 31st AAAI Conference on Artificial Intelligence (AAAI), pp. 642-648.

Peters, D. (2018). Single-peakedness and total unimodularity: New polynomial-time algorithms for multi-winner elections. In Proceedings of the 32nd AAAI Conference on Artificial Intelligence (AAAI), pp. 1169-1176.

Peters, D., \& Elkind, E. (2016). Preferences single-peaked on nice trees. In Proceedings of the 29th AAAI Conference on Artificial Intelligence (AAAI), pp. 594-600.

Peters, D., \& Lackner, M. (2017). Preferences single-peaked on a circle. In Proceedings of the 31st AAAI Conference on Artificial Intelligence (AAAI), pp. 649-655.

Rothe, J., Spakowski, H., \& Vogel, J. (2003). Exact complexity of the winner problem for Young elections. Theory of Computing Systems, 36(4), 375-386.

Sato, S. (2010). Circular domains. Review of Economic Design, $14(3-4), 331-342$.

Schrijver, A. (1998). Theory of linear and integer programming. John Wiley \& Sons.

Schummer, J., \& Vohra, R. V. (2002). Strategy-proof location on a network. Journal of Economic Theory, 104(2), 405-428.

Seymour, P. (1980). Decomposition of regular matroids. Journal of Combinatorial Theory, Series B, 28(3), 305-359.

Skowron, P. (2015). What do we elect committees for? A voting committee model for multi-winner rules. In Proceedings of the 24th International Conference on Artificial Intelligence (IJCAI), pp. 1141-1147.

Skowron, P., Faliszewski, P., \& Lang, J. (2016). Finding a collective set of items: From proportional multirepresentation to group recommendation. Artificial Intelligence, 241, 191-216.

Sui, X., Francois-Nienaber, A., \& Boutilier, C. (2013). Multi-dimensional single-peaked consistency and its approximations. In Proceedings of the 23rd International Joint Conference on Artificial Intelligence (IJCAI), pp. 375-382.

Szufa, S., Faliszewski, P., Skowron, P., Slinko, A., \& Talmon, N. (2020). Drawing a map of elections in the space of statistical cultures. In Proceedings of the 19th International Conference on Autonomous Agents and Multiagent Systems (AAMAS). To appear.

Tangian, A. (2020). Policy representation across the political spectrum. In Analytical Theory of Democracy, pp. 405-446. Springer.

Tardos, E. (1986). A strongly polynomial algorithm to solve combinatorial linear programs. Operations Research, 34(2), 250-256. 
Waton, S. D. (2007). On permutation classes defined by token passing networks, gridding matrices and pictures: three flavours of involvement. Ph.D. thesis, University of St Andrews.

Yu, L., Chan, H., \& Elkind, E. (2013). Multiwinner elections under preferences that are single-peaked on a tree. In Proceedings of the 23rd International Joint Conference on Artificial Intelligence (IJCAI), pp. 425-431.

Zou, S., \& Li, M. (2015). Facility location games with dual preference. In Proceedings of the 14th International Conference on Autonomous Agents and Multiagent Systems (AAMAS), pp. 615-623. 